\title{
Photoexcitation and ionization in molecular oxygen: Theoretical studies of electronic transitions in the discrete and continuous spectral intervals
}

\author{
A. Gerwer, C. Asaro, and B. V. McKoy \\ A. A. Noyes Laboratory of Chemical Physics, California Institute of Technology, Pasadena, \\ California 91125

\section{P. W. Langhoff} \\ Department of Chemistry, Indiana University, Bloomington, Indiana 47401, ${ }^{\text {a) }}$ \\ Department of Aeronautics and Astronautics, Stanford University, Stanford, California 94305 , \\ and Computational Chemistry Group, NASA-Ames Research Center, Moffett Field, California 94035 \\ (Received 16 April 1979; accepted 27 April 1979)
}

\begin{abstract}
Theoretical studies of valence-electron $\left(1 \pi_{g}, 1 \pi_{u}, 3 \sigma_{g}\right)$ photoexcitation and ionization cross sections in molecular oxygen are reported employing separated-channel static-exchange calculations and the Stieltjes-Tehebycheff moment-theory technique. As in previously reported investigations of photoexcitation and ionization in small molecules following this approach, canonical Hartree-Fock orbitals, large Gaussian basis sets, and many-electron eigenstates of correct symmetry are used in defining appropriate noncentral static-exchange potentials and in computations of the appropriate discrete and continuum transition strengths. It is particularly important in molecular oxygen to incorporate the appropriate ionic parentages of the various photoionization multiplet states in order to obtain the correct partial-channel cross sections. The calculated discrete series associated with $1 \pi_{g}$ excitation are found to be in good agreement with available experimental assignments and previously reported theoretical studies, and the predicted states associated with $1 \pi_{u}$ and $3 \sigma_{g}$ excitations are in general accord with assignments for the higher series based on spectral and quantum-defect analysis. Although the observed photoelectron spectra and photoionization cross sections are relatively complex, the calculated total vertical electronic photoabsorption cross section and the partial-channel photoionization cross sections for production of $X^{2} \Pi_{g}, a^{4} \Pi_{u}, A^{2} \Pi_{u}, 2^{2} \Pi_{u}, 3^{3} \Pi_{u}, b^{4} \Sigma_{g}^{-}$, and $B^{2} \Sigma_{g}^{-}$ionic states are found to be in good accord with recent synchrotron radiation, line-source, electron-impact, and $(e, 2 e)$ dipole oscillator-strength measurements when proper account is taken of the parentages of the various multiplet states. The partial-channel cross sections exhibit resonancelike structures that can be attributed to contributions from diabatic valencelike virtual states that appear in the appropriate photoionization continua, rather that in the corresponding discrete spectral intervals. These features in the dipole spectrum of molecular oxygen are discussed and are contrasted and compared with the results of previously reported related studies in molecular nitrogen and carbon monoxide.
\end{abstract}

\section{INTRODUCTION}

Recently reported theoretical ${ }^{1-6}$ and experimental ${ }^{7-14}$ photoexcitation and ionization studies reveal the presence of resonancelike features in certain of the partial-channel photoionization cross sections of diatomic $\left(\mathrm{N}_{2}, \mathrm{CO}\right)$ and polyatomic $\left(\mathrm{H}_{2} \mathrm{O}, \mathrm{H}_{2} \mathrm{CO}\right)$ molecules. Elementary qualitative arguments suggest these resonance features can be attributed to contributions to the cross sections from $\sigma^{*}$ and other valencelike virtual orbitals that appear in the appropriate photoionization continua, rather than in corresponding discrete spectral intervals. To achieve quantitative agreement with the measured outervalence-shell partial-channel cross sections, it is apparently sufficient to employ the static-exchange approximation and noncentral molecular potentials in the calculations. Appropriately chosen local noncentral potentials can also reproduce some of the general features of the spectra, ${ }^{15,16}$ although simpler approxifhations involving, for example, plane-wave ${ }^{17}$ and one- and twocenter ${ }^{18,19}$ Coulombic final states generally lead to qualitatively incorrect cross sections that can be an order of magnitude or more in error. In view of the fundamental importance of resonances in molecular photoion-

\footnotetext{
2) Permanent address.
}

ization continua to our understanding of electronic excitation/ionization spectra and their molecular-orbital interpretations, and in light of continuing line-source, synchrotron and bremsstrahlung radiation, electronimpact, and $(e, 2 e) /(e, e+$ ion) coincidence studies of dipole processes in small molecules, further quantitatively reliable theoretical investigations of appropriately chosen systems are clearly in order.

The photoabsorption spectrum of molecular oxygen has been the subject of continuing experimental and the oretical interest, as a partial consequence of its impor tance in atmospheric and related photochemistry. ${ }^{20}$ In spite of a great many previous theoretical and experimental investigations, ${ }^{21}$ the general features of the oxygen continuous spectrum have been experimentally established comparatively recently, ${ }^{22-28}$ and the reported corresponding theoretical studies do not particularly clarify the observed results. In the present study, outer-valence-shell electronic photoexcitation and ionization cross sections in molecular oxygen are obtained in the separated-channel static-exchange approximation employing the previously devised Stieltjes-Tchebycheff (S-T) moment-theory technique and $L^{2}$ variational methods. ${ }^{29-32}$ As in previously reported studies of photoexcitation and ionization employing this approach, ${ }^{1-6} \mathrm{Har}-$ 
tree-Fock canonical orbitals and Gaussian basis functions are used in molecular oxygen in calculating one-electron discrete excitation series and continuum pseudostate spectra, and many-electron eigenstates of correct symmetry are employed in calculations of appropriate transition strengths. Particular care must be exercised in properly incorporating the ionic parentages of the various multiplet photoionization states in order to obtain the correct partial-channel cross sections in molecular oxygen. Excitations of the $1 \pi_{\ell}, 1 \pi_{u}$, and $3 \sigma_{g}$ outer valence orbitals are considered, whereas studies of the $2 \sigma_{z}, 2 \sigma_{u}$ inner-valence and $1 \sigma_{p}, 1 \sigma_{u} K$-shell orbitals are described in separate reports. The calculated discrete series converging on the ground $\left(1 \pi_{g}^{-1}\right) X^{2} \Pi_{g}$ molecular ionic state are found to be in generally good agreement with experimental assignments and previously reported theoretical values, in spite of the use of a frozen core and the single-configurational approximation. Although the predicted positions of the higher $1 \pi_{u}, 3 \sigma_{g}$ excitation series are in general accord $w$ ith assignments made on basis of spectral and quantum-defect analysis, definitive studies in these regions will require appropriate autoionizing line-shape calculations not reported here. The calculated partial-channel cross sections for the production of $X^{2} \Pi_{p}, a^{4} \Pi_{u}, A^{2} \Pi_{u}, 2^{2} \Pi_{u}, 3^{2} \Pi_{u}, b^{4} \Sigma_{c}^{-}$and $B^{2} \Sigma_{\text {in }}^{-}$ionic states are found to be in good accord with recent line-source $\mathrm{e}^{25}$ and $(e, 2 e)^{28}$ dipole oscillator-strength measurements. Similarly, the calculated total photoabsorption/ionization cross section is found to be in good accord with the results of recent electron impact, ${ }^{22,28}$ continuum and line source, ${ }^{23-26}$ and $(e, e+\text { ion })^{28}$ dipole oscillator-strength measurements. Although the measured photoelectron spectra and corresponding partialchannel and total photoabsorption/ionization cross sections in molecular oxygen are relatively complex, the static-exchange approximation is seen to account satisfactorily for the observed results, provided proper cognizance is taken of the parentages of the various multiplet states. Moreover, a previously unassigned band in the photoelectron/ionization spectrum is assigned on basis of the present investigation. Resonance features appearing in certain of the individual channel components are attributed to contributions to the cross sections from valencelike diabatic states appearing in the corresponding photoionization continua. Specifically, compact $\sigma_{u} 2 p, \pi_{u} 3 p, \pi_{u} 3 d, \delta_{\varepsilon} 3 d$, and $\delta_{u} 3 d$ orbitals apparently contribute to the $1 \pi_{e}^{-1}, 1 \pi_{u}^{-1}$, and $3 \sigma_{q}^{-1}$ valence photoionization channels, rather than to the corresponding discrete spectral intervals. These and other features of the molecular oxygen spectrum are discussed, and are contrasted and compared with the results of related previously reported theoretical investigations of dipole excitation/ionization spectra in molecular nitrogen and carbon monoxide. ${ }^{1-3}$

A brief description of the Stieltjes-Tchebycheff technique in the separated-channel static-exchange approximation is given in Sec. II, the calculations required in molecular oxygen are summarized in Sec. III, and the resulting photoexcitation and ionization cross sections are reported in Sec. IV. A summary discussion of the resonance features is provided in Sec. $V$ and concluding remarks are made in Sec. VI.

\section{THEORETICAL APPROACH}

The Stieltjes-Tchebycheff procedure and the separated-channel static-exchange approximation are described in considerable detail in previous publications. ${ }^{1-6,29-32}$ Consequently, it is sufficient here to provide a brief heuristic description of the general scheme, and to refer the reader to earlier reports for further details.

A ground-state Hartree-Fock function is first constructed near the equilibrium molecular geometry employing Gaussian basis orbitals and appropriate computational methods. ${ }^{33,34}$ Many-electron eigenstates of correct parentage and symmetry are employed in construct ing noncentral static-exchange potentials for each orbital excitation considered from the occupied canonical orbitals, and corresponding one-electron Schrödinger equations are formed for the calculation of excitation spectra. The one-electron equations are solved variationally employing significantly larger basis sets than that used in constructing the ground-state Hartree-Fock function. A considerable reduction in computational ef fort is achieved by noting that only a subset of two-electron integrals, involving two basis orbitals from the occupied valence space and two from the supplemental or virtual space, is required in constructing variational solutions. ${ }^{1,2}$ The one-electron eigenvalues obtained that are below the occupied-orbital ionization potential, and the corresponding one-electron orbitals, provide approximations to the appropriate discrete or autoionizing valence and Rydberg states. Those eigenval ues above the ionization potential, and their corresponding orbitals, provide pseudospectra for the Stieltjes-Tchebycheff moment analysis, from which the appropriate photionization cross sections are obtained. The necessary excitation and ionization transition strengths are constructed from many-electron eigenstates of correct parentage and symmetry. It is important to note that in order for the $S-T$ analysis to be satisfactory, and to provide the correct static-exchange photoionization cross sections, it is necessary that the calculated pseudospectra correctly describe the appropriate continuum eigenstates over finite regions of space.

The S-T approach employs a pseudospectrum of discrete transition frequencies and oscillator strengths, calculated in the present study as indicated above, in a smoothing procedure in which a continuous approximation to the underlying photoionization cross section is obtained. Conventional spectral power moments, which are convergent in the $L^{2}$ pseudospectrum of energies and strengths, ${ }^{35}$ are generally regarded as intermediaries in the smoothing procedure, providing a basis for establishing the convergence of the overall procedure. In actual calculations, however, the power moments are avoided, and a highly stable computational algorithm is employed in constructing so-called recurrence coefficients, and moment-theory spectra of (principal) frequency points and weights, directly from the quantummechanically determined pseudospectrum. ${ }^{20-32}$ The moment-theory spectra exhibit usedful properties which the original pseudospectrum does not exhibit, and, in particular, provide bounds on the cumulative oscillator- 
strength distribution. The latter can be differentiated in a variety of ways to provide a final expression for the photoionization cross section. Stieltjes derivatives of various orders are generally employed to obtain a first approximation to the continuous cross section, and to establish the range of convergence of the variationally calculated recurrence coefficients. Tchebycheff derivatives and appropriate recurrence-coefficient extention procedures are then used to construct a continuous approximation to the cross section. Alternatively, specific analytic forms fit to the cumulative Stieltjes histograms provide expressions convenient for differentiation. Previous investigations indicate the method is highly satisfactory, provided that the quantum-mechanical pseudostate calculation contains sufficient information pertaining to the underlying continuous spectrum. ${ }^{1-6}$

It is perhaps appropriate to note that the $\mathrm{S}-\mathrm{T}$ approach to photoionization is not limited to the separated-channel static-exchange approximation employed here. Coupledchannel static-exchange calculations, for example, can also be performed employing generally available computer codes. Since a number of static ionic cores are employed in this approach, well-defined total and partial-channel cross sections are obtained directly from the calculations. By contrast, when correlated groundstate functions and polarized or relaxed final-state core functions are employed, special projection or related procedures - which have yet to be clarified fully - are required in determining the pseudospectra corresponding to specific photoionization channels. Moreover, the necessary quantum-mechanical calculations, of course, become somewhat more elaborate when correlated ground-state and ionic core function are employed. In order to gain further experience with the S-T approach to molecular photoionization, and to clarify the general natures of molecular photoionization cross sections, it is perhaps appropriate to investigate members of the large class of molecules for which the static-exchange approximation is suitable. This class should include those molecules in which photoelectron spectra are well described by the orbital approximation and Koopmans' theorem, and in which the effects of shake up and rearrangement are small. Although the observed valence photoelectron ${ }^{36-38}$ and photoionization ${ }^{25-28}$ spectra in molecular oxygen are relatively complex, it will be seen that the static-exchange approximation accounts satisfactorily for the measured results provided proper cognizance is taken of the ionic parentages of the various multiplet states. Relaxation and related effects are presumably of more consequence in the inner-valence and core spectra, however. ${ }^{22-24}$

\section{MOLECULAR OXYGEN CALCULATIONS}

The electronic ground state of molecular oxygen is written in the Hartree-Fock approximation in the symbolic form

$$
\left(1 \sigma_{s}^{2} 1 \sigma_{u}^{2} 2 \sigma_{s}^{2} 2 \sigma_{u}^{2} 3 \sigma_{l}^{2} 1 \pi_{u}^{4} 1 \pi_{g}^{2}\right) X^{3} \Sigma_{\varepsilon}^{-},
$$

where the indicated canonical orbitals are obtained in the conventional manner. ${ }^{33,34}$ In the present calculations a $(10 s, 5 p, 1 d) /[3 s, 2 p, 1 d]$ contracted Gaussian basis set is employed at $R_{e}=2.282 a_{0}$, giving a total
SCF energy of -149.634 a.u., ${ }^{34}$ compared with the $\mathrm{H}-\mathrm{F}$ limit of -149.666 a.u. ${ }^{39}$

The types of one-electron excitation and ionization series considered here can be written in the symbolic forms

$$
\begin{aligned}
& \left(1 \pi_{g}^{-1} k \sigma_{u}\right)^{3} \Pi_{u},\left(1 \pi_{\varepsilon}^{-1} k \pi_{u}\right)^{3} \Sigma_{u}^{-},\left(1 \pi_{g}^{-1} k \delta_{u}\right)^{3} \Pi_{u}, \\
& \left(1 \pi_{u}^{-1} k \sigma_{q}\right)^{3} \Pi_{u},\left(1 \pi_{u}^{-1} k \pi_{q}\right)^{3} \Sigma_{u}^{-},\left(1 \pi_{u}^{-1} k \delta_{\varepsilon}\right)^{3} \Pi_{u}, \\
& \left(3 \sigma_{g}^{-1} k \sigma_{u}\right)^{3} \Sigma_{u}^{-},\left(3 \sigma_{g}^{-1} k \pi_{u}\right)^{3} \Pi_{u},
\end{aligned}
$$

where excitations of the $2 \sigma_{q}, 2 \sigma_{u}$ and $1 \sigma_{z}, 1 \sigma_{u}$ orbitals, which remain doubly occupied, are not considered. There are, consequently, eight distinct individual channels for excitation of the $3 \sigma_{e}, 1 \pi_{u}$, and $1 \pi_{e}$ orbitals, three corresponding to ${ }^{3} \Sigma_{u}^{-}$symmetry, and five to ${ }^{3} \Pi_{u}$ symmetry. However, because of the open $1 \pi_{z}$ shell in $\mathrm{O}_{2}$ some of the eight channels are comprised of two or more subchannels associated with the formation of various distinct parent molecular $\mathrm{O}_{2}^{+}$ions. Appropriate many-electron pseudospectral wave functions and corresponding static-exchange potentials must be constructed for each of these subchannels.

In the case of excitation and ionization of the outer most $1 \pi_{g}$ orbital [Eq. (2a)], the resulting core configuration gives rise to only the ground $X^{2} \Pi_{f}$ molecular $\mathrm{O}_{2}^{+}$ ionic state. Consequently, in this case three appropriate sets of many-electron pseudospectral functions and three corresponding static-exchange potentials must be constructed to describe the excitation and ionization of $1 \pi_{\ell}$ electrons into $k \sigma_{u}, k \pi_{u}$, and $k \delta_{u}$ orbitals, respectively. In the case of excitation and ionization of the $1 \pi_{u}$ orbital [Eq. (2b)], however, the remaining $1 \pi_{u}^{3} 1 \pi_{q}^{2}$ core configuration gives rise to five separate electronic molecular ionic states. Of these, two $\left[a^{4} \Pi_{u},{ }^{2} \Pi_{u}(1)\right]$ arise from the ground-state $\left(1 \pi_{g}^{2}\right)^{3} \Sigma_{c}^{-}$parent, whereas three $\left[{ }^{2} \Phi_{u},{ }^{2} \Pi_{u}(2),{ }^{2} \Pi_{u}(3)\right]$ arise from the excited-state $\left(1 \pi_{f}^{2}\right)^{1} \Sigma_{g}^{+}$and $\left(1 \pi_{\varepsilon}^{2}\right)^{1} \Delta_{f}$ parents. As a consequence of the frozen-core approximation inherent in the static-exchange approach, only the $a^{4} \Pi_{u}$ and ${ }^{2} \Pi_{u}(1)$ ionic states contribute to dipole transitions in molecular oxygen, since these arise from the $\left(1 \pi_{e}^{2}\right)^{3} \Sigma_{z}^{-}$parent. However, approximations to the spectroscopically observed $A^{2} \Pi_{w}$, $2^{2} \Pi_{u}$, and $3^{2} \Pi_{w} \mathrm{O}_{2}^{+}$states ${ }^{40}$ are formed from linear combinations of the ${ }^{2} \Pi_{u}(1),{ }^{2} \Pi_{u}(2)$, and ${ }^{2} \Pi_{u}(3)$ static-exchange states in the present development. Consequently, four parent $\mathrm{O}_{2}^{+}$ionic states $\left[a^{4} \Pi_{u}, A^{2} \Pi_{u}, 2^{2} \Pi_{u}, 3^{2} \Pi_{u}\right]$ can be produced on basis of the static-exchange approach upon excitation and ionization of a $1 \pi_{u}$ electron. Strictly speaking, it is necessary to construct distinct $1 \pi_{u}-k \sigma_{g}$, $k \pi_{s}, k \delta_{s}$ orbital pseudospectra corresponding to each of these four separate ionic states. However, in accordance with the approximations inherent in multiplet theory, virtual orbitals are chosen here in a democratic fashion to be appropriate for all the states arising from $1 \pi_{u}^{3} 1 \pi_{\varepsilon}^{2} k \sigma_{g}, 1 \pi_{u}^{3} 1 \pi_{g}^{2} k \pi_{\varepsilon}$, and $1 \pi_{u}^{3} 1 \pi_{g}^{2} k \delta_{g}$ configurations. In this way only three distinct orbital pseudospectra are required in forming the 12 many-electron pseudospectra associated with the four parent $\mathrm{O}_{2}^{+}$ionic states formed upon excitation and ionization of a $1 \pi_{u}$ electron. In the case of $3 \sigma_{q}$ excitations [Eq. (2c)], the $3 \sigma_{p} 1 \pi_{p}^{2}$ configuration gives rise to $b^{4} \Sigma_{q}^{-}, B^{2} \Sigma_{k}^{-},{ }^{2} \Sigma_{k}^{+}$, and $C^{2} \Delta_{q}$ final $\mathrm{O}_{2}^{+}$ 
TABLE I. Static-exchange open-shell potentials in molecular oxygen. ${ }^{\mathrm{a}, \mathrm{b}}$

\begin{tabular}{rlllll}
\hline \hline \multicolumn{1}{r}{} & $3 \sigma_{g}$ & $1 \pi_{u}^{x}$ & $1 \pi_{u}^{y}$ & $1 \pi_{g}^{x}$ & $1 \pi_{g}^{y}$ \\
\hline $3 \sigma_{g} \rightarrow k \sigma_{u}$ & $\frac{1}{2} /-1$ & $1 / 1$ & $1 / 1$ & $\frac{1}{2} / \frac{1}{2}$ & $\frac{1}{2} / \frac{1}{2}$ \\
$\rightarrow k \pi_{u}^{x}$ & $\frac{1}{2} /-1$ & $1 / 1$ & $1 / 1$ & $\frac{1}{2} / \frac{1}{2}$ & $\frac{1}{2} / \frac{1}{2}$ \\
$1 \pi_{u}^{x} \rightarrow k \sigma_{g}$ & $1 / 1$ & $\frac{3}{4} / \frac{1}{2}$ & $\frac{3}{4} / \frac{1}{2}$ & $\frac{1}{2} / \frac{1}{2}$ & $\frac{1}{2} / \frac{1}{2}$ \\
$\rightarrow k \pi_{g}^{x}$ & $1 / 1$ & $\frac{1}{2} /-3$ & $1 / 5$ & $\frac{1}{2} / \frac{1}{2}$ & $\frac{1}{2} / \frac{1}{2}$ \\
$\rightarrow k \delta_{g}^{x y}$ & $1 / 1$ & $\frac{3}{4} / \frac{1}{2}$ & $\frac{3}{4} / \frac{1}{2}$ & $\frac{1}{2} / \frac{1}{2}$ & $\frac{1}{2} / \frac{1}{2}$ \\
$1 \pi_{g}^{x} \rightarrow k \sigma_{u}$ & $1 / 1$ & $1 / 1$ & $1 / 1$ & $\frac{1}{4} / \frac{1}{2}$ & $\frac{1}{4} / \frac{1}{2}$ \\
$\rightarrow k \pi_{u}^{x}$ & $1 / 1$ & $1 / 1$ & $1 / 1$ & $-\frac{1}{4} /-1$ & $\frac{3}{4} / 3$ \\
$\rightarrow k \delta_{u}^{x y}$ & $1 / 1$ & $1 / 1$ & $1 / 1$ & $-\frac{1}{4} /-1$ & $\frac{3}{4} / 3$ \\
\hline \hline
\end{tabular}

${ }^{2}$ Values of the coefficients $a_{i}^{\Gamma} / b_{i}^{\Gamma}$ appearing in the potential of Eq. (5), determined employing the procedures of J. B. Rose and V. McKoy, J. Chem. Phys. 55, 5435 (1971).

'The $1 \sigma_{g}, 1 \sigma_{u}$ and $2 \sigma_{g}, 2 \sigma_{u}$ orbitals remain doubly occupied and, consequently, $a_{i}^{\Gamma}=1, b_{i}^{\Gamma}=1$ in these cases.

ionic states. Of these, only $b^{4} \Sigma_{\varepsilon}^{-}$and $B^{2} \Sigma_{c}^{-}$, which arise from the $\left(1 \pi_{p}^{2}\right)^{3} \Sigma_{p}^{-}$ground -state configuration, contribute to the molecular oxygen excitation and ionization spectra in the static-exchange approximation. As in the case of the $1 \pi_{u}$ orbital, democratically chosen $k \sigma_{u}$ and $k \pi_{u}$ or bitals appropriate for both ionic states are employed in constructing many-electron wave functions for $3 \sigma_{q}$ excitation and ionization. More refined calculations in which orbitals associated with specific final ionic states are employed can, of course, also be performed. It is found, however, that the pseudospectral orbitals are relatively insensitive to these refinements, and, consequently, democratically chosen orbitals are employed here.

In each of the eight individual cases [Eqs. (2)], the appropriate one-electron Schrödinger equations are written as

$$
\left(h_{\Gamma}-\epsilon\right) \phi_{\Gamma, 6}=0,
$$

where $\epsilon$ corresponds to continuum or discrete energies, and

$$
h_{\Gamma}=T+V+V_{\Gamma}
$$

is the static-exchange Hamiltonian, with $T$ and $V$ the kinetic and nuclear-framework-potential energy operators, and $V_{\Gamma}$ the appropriate nonlocal channel potential. The nonlocal molecular channel potentials $V_{\Gamma}$ are all written in the forms

$$
V_{\Gamma}=\sum_{i} 2 a_{i}^{\Gamma} J_{i}-b_{i}^{\Gamma} K_{i}
$$

where $J_{i}$ and $K_{i}$ are the familiar orbital Coulomb and exchange operators, ${ }^{33}$ and the coefficients $a_{i}^{\Gamma}, b_{i}^{\Gamma}$ must be determined in each case. Explicit expressions for the latter, based on a previously reported analysis of openshell SCF energy expressions, ${ }^{41}$ are given in Table $\mathrm{I}$. It is perhaps helpful to note in this connection that the static-exchange calculations give results identical with dipole spectra obtained from appropriate single-excitation configuration-interaction calculations in which $\mathrm{Har}$ -
tree-Fock ground-state canonical orbitals are used to describe the frozen final-state core configu rations. ${ }^{42}$ Consequently, the open-shell static-exchange potentials of Table I are obtained simply by including in the appropriate energy functionals multiplicity weighted contribu tions from all linearly independent eigenstates of correct symmetry that can be formed by coupling the photoexcited final-state orbitals with the appropriate static core states indicated above.

In order to obtain useful variational solutions of Eq. (3) in each case, it is necessary to use relatively large basis sets of Gaussian orbitals. This large-basis-set requirement is a partial consequence of the structure of Cartesian Gaussian functions, which exhibit only the minimum number of nodes for each orbital angular momentum considered. Fortunately, it is not necessary that the variational solutions reproduce in detail the oscillatory behaviors of scattering functions over large regions of space in order to obtain reliable photoionization cross sections, since the product of dipole moment operator and ground state orbital provides a finite box in which the final-state function must be determined. ${ }^{32}$ Even with this helpful simplification, however, it is generally necessary to expand the basis sets employed to the limit of linear dependence in order to obtain fully converged results.

In Table II are shown the supplemental basis functions employed in the present study in addition to the valence basis used in construction of the ground-state HartreeFock function. ${ }^{34}$ The variational solutions of Eq. (3) obtained in this extended basis correspond to pseudospectra of $23 \sigma_{s}, 23 \sigma_{u}, 16 \pi_{s}, 16 \pi_{u}, 13 \delta_{z}$, and $9 \delta_{u}$ orbitals. As a convenience in evaluating the appropriate transition integrals, the solutions of Eq. (3) are explicitly orthogonalized to the occupied canonical Fock orbitals. Past experience indicates this generally has small effect on the calculated cross sections relative to results obtained without the explicit orthogonality constraint. In the case of molecular oxygen, however, there is a somewhat more significant effect in that the $1 \pi_{u} \rightarrow 1 \pi_{g}$

\begin{tabular}{|c|c|c|c|}
\hline Location & Type & Number & Exponent range $^{b}$ \\
\hline \multicolumn{4}{|c|}{$\left(\sigma_{g} / \sigma_{u}\right)$ symmetry } \\
\hline $0 / 0$ & $s / s$ & $4 / 4$ & $2.0-0.12 / 0.0862-0.0024$ \\
\hline $0 / 0$ & $p_{z} / p_{z}$ & $4 / 4$ & $2.0-0.12 / 0.0637-0.00169$ \\
\hline c.m. $/ \mathrm{c} \cdot \mathrm{m}$. & $s / p_{z}$ & $1.2 / 12$ & $0.2-0.00052 / 0.2-0.00052$ \\
\hline \multicolumn{4}{|c|}{$\left(\pi_{g} / \pi_{u}\right)$ symmetry } \\
\hline $0 / 0$ & $p_{x} / p_{x}$ & $4 / 4$ & $0.7-0.6 / 0.7-0.06$ \\
\hline $0 / 0$ & $d_{x z} / d_{x z}$ & $4 / 4$ & $0.7-0.1 / 0.7-0.06$ \\
\hline c.m. /c.m. & $d_{x z} / p_{x}$ & $6 / 6$ & $0.5-0.025 / 0.025-0.001$ \\
\hline \multicolumn{4}{|c|}{$\left(\delta_{\mathfrak{g}} / \delta_{u}\right)$ symmetry } \\
\hline $0 / 0$ & $d_{x y} / d_{x y}$ & $5 / 9$ & $0.5-0.1 / 0.5-0.005$ \\
\hline c.m. $/ \mathrm{c} . \mathrm{m}$. & $d_{x y} / \cdots$ & $8 / \cdots$ & $0.2-0.007 / \cdots$ \\
\hline
\end{tabular}

TABLE II. Supplemental Gaussian basis for molecular oxygen calculations, a

aSupplemental basis functions employed, in addition to the $(10 \mathrm{~s}$, $5 p, 1 d) /[3 s, 2 p, 1 d]$ valence basis, in solution of Eq. (3). bRatios of successive exponents vary geometrically. 
TABLE III. $1 \pi_{k}$ excitation spectra in $\mathrm{O}_{2}$ (I. P. $=12.1 \mathrm{eV}$ ).

\begin{tabular}{|c|c|c|}
\hline $\begin{array}{l}\text { Present results } \\
\text { Energy }(\mathrm{eV}) / f \text { Number }\end{array}$ & $\begin{array}{l}\text { Experimental values } \\
\text { Energy }(e V) / \text { Intensity }\end{array}$ & $\begin{array}{l}\text { Previous calculations } \\
\text { Energy (eV)/f Number }\end{array}$ \\
\hline \multicolumn{3}{|c|}{$\left(1 \pi_{g}\right)^{3} \Sigma_{z}^{*}-\left(n p \pi_{u}\right)^{3} \Sigma_{u}^{*}$} \\
\hline $9.42 / 0.00271$ & 9.31/weak & $9.44 / 0.00215$ \\
\hline $10.82 / 0.00076$ & 10.46 & $10.85 / 0.00073$ \\
\hline $11.34 / 0.00032$ & 11.36 & $11.32 / 0.00033$ \\
\hline $11.59 / 0.00016$ & 11.54 & $\cdots$ \\
\hline $11.76 / \cdots$ & 11.62 & $\cdots$ \\
\hline \multicolumn{3}{|c|}{$\left(1 \pi_{g}\right)^{3} \Sigma_{g}^{-} \rightarrow\left(n p \sigma_{u}\right)^{3} \Pi_{u}$} \\
\hline $8.53 / 0.00000$ & $\cdots$ & $7.99 / \cdots$ \\
\hline $10.16 / 0.00835$ & $9.97 /$ strong & $9.98 / 0.00833$ \\
\hline $11.00 / 0.00549$ & 10.98 & $10.96 / 0.00376$ \\
\hline $11.41 / 0.00285$ & 11.36 & $11.32 / 0.00201$ \\
\hline $11.63 / 0.00160$ & 11.54 & $\cdots$ \\
\hline $11.75 / 0.00099$ & 11.62 & $\cdots$ \\
\hline $11.86 / \cdots$ & $\cdots$ & $\cdots$ \\
\hline \multicolumn{3}{|c|}{$\left(1 \pi_{g}\right)^{3} \Sigma_{g}^{-}-\left(n \delta_{u}\right)^{3} \Pi_{u}$} \\
\hline $11.22 / 0.00015$ & $\cdots$ & $10.68 / \cdots$ \\
\hline $11.74 / \cdots$ & $\cdots$ & 11.41 \\
\hline
\end{tabular}

Values obtained from Eqs. (3) to (6) and appropriate multiplet line-strength factors employing the basis sets described in Sec. III and Table II. The indicated series are made to converge on the experimentally determined $X^{2} \Pi_{g}$ ionic state, as discussed in the text.

Spectral assignments and intensities taken from $Y$. Tanaka and T. Takamine, Tokyo Inst. Phys. Chem. Res. 39, 437 (1942); E. Lindholm, Ark. Fys. 40, 117 (1969). See also the tabulations of P. H. Krupenie, J. Phys. Chem. Ref. Data 1, 423 (1972), and references cited therein.

'Previously reported IVO calculations of D. C. Cartwright, W. J. Hunt, W. Williams, S. Trajmar, and W. A. Goddard, J. Chem. Phys. 8, 2436 (1973). Additional theoretical calculations are reported by J. LeClereq, Ann. A strophys. 30, 93 (1967); T. Betts and V. McKoy, J. Chem. Phys, 54, 113 (1971); H. F. Schaefer and W. H. Miller, J. Chem. Phys. 55, 4107 (1971); K. Morokuma and H. Konishi, J. Chem. Phys. 55, 402 (1971); R. J. Buenker and S. D. Peyerimhoff, Chem. Phys. 8, 324 (1975); Chem. Phys. Lett. 34, 225 (1975); R. P. Saxon and B. Liu, J. Chem. Phys. 67, 5432 (1977). The values cited for the $1 \pi_{g} \rightarrow n \delta_{u}$ series are obtained from the Rydberg formula for $n=3$ and 4 employing a quantum defect of 0.1 . Estimates for $f$ numbers obtained from the Coulomb approximation (Ref. 35 ).

transition is excluded from the calculation by the orthogonality constraint. Since this excitation in the staticexchange approximation corresponds to the well-known $X^{3} \Sigma_{z}^{-}-B^{3} \Sigma_{u}^{-}$Schmann-Runge bands, ${ }^{40}$ however, it is not necessary to explicitly add the appropriate vertical energy and $f$ number to the spectrum in this case.

As in the previously reported studies in this series, ${ }^{1-3}$ one-electron transition energies and oscillator strengths

$$
\begin{aligned}
& \bar{\epsilon}_{i}^{\Gamma}=\epsilon_{\Gamma}+\epsilon_{i}^{\Gamma}, \\
& \bar{f}_{i}^{\Gamma}=(2 / 3) \bar{\epsilon}_{i}^{\Gamma}\left|\left\langle\phi_{i}^{\Gamma}|\mu| \phi_{\Gamma}\right\rangle\right|^{2}
\end{aligned}
$$

are formed using experimental, rather than Koopmans, adiabatic ionization potentials $\epsilon_{r},{ }^{36-38}$ thereby insuring convergence to the appropriate series limits, and allowing in some measures for the effects of core relaxation. When the one-electron pseudospectra of Eqs. (6) are determined for the eight individual channels of Eqs. (2) to (5) and Table I, it only remains to determine the total line strengths associated with each of the many-electron final states. ${ }^{43,44}$ These provide the many-electron pseudospectra of transition frequencies and multiplet oscillator strengths associated with the production of specific parent molecular $\mathrm{O}_{2}^{+}$ionic states.

The foregoing analysis indicates that the static-exchange approach to valence -electron $\left(1 \pi_{\varepsilon}, 1 \pi_{u}, 3 \sigma_{\varepsilon}\right)$ photoionization in molecular oxygen predicts the appearance of seven distinct ionic states $\left[X^{2} \Pi_{f}, a^{4} \Sigma_{\psi}^{-}, A^{2} \Pi_{u}, b^{4} \Sigma_{g}^{-}\right.$, $\left.B^{2} \Sigma_{z}^{-}, 2^{2} \Pi_{u}, 3^{2} \Pi_{u}\right]$ in the photoelectron spectra. These are all observed in the $(e, 2 e)$ experiments, ${ }^{28}$ although some of the states are not separately resolved. Higherresolution lower-energy line-source measurements ${ }^{25,36-38}$ are helpful in these cases in separating the unresolved $(e, 2 e)$ data. The additional ionic states observed in the $(e, 2 e)$ spectra $\left[c^{4} \Sigma_{u}^{-},{ }^{2} \Sigma_{u}^{-},{ }^{2,4} \Sigma_{e}^{-}\right]$can be attributed to $2 \sigma_{u}$ and $2 \sigma_{g}$ photoionization not treated here, with the exception of the $c^{4} \Sigma_{u}^{-}$state, which is a well-known two-electron excitation. ${ }^{45}$ The latter contributes weakly to the photoelectron and ionization spectra, however, and, consequently, the static-exchange approximation apparently accounts for all the important observed features of the valence-shell measurements.

\section{PHOTOEXCITATION AND IONIZATION CROSS SECTIONS IN MOLECULAR OXYGEN}

In Tables III-V and Figs. 1-4 are shown the discrete excitation series and photoionization cross sections, respectively, obtained from the present calculations. These results are discussed in detail in the following subsections. In order to obtain some preliminary qualitative indication of the natures of the spectra, it is perhaps helpful to recall that both compact and diffuse virtual molecular orbitals are anticipated in the excitation spectrum of molecular oxygen on basis of elementary considerations. ${ }^{46,47}$ Specifically, a valencelike $\sigma_{u} 2 p$ or $\sigma^{*}$ virtual orbital is expected to participate in strong $N-V_{\sigma}$ excitations in the molecular oxygen spectrum, and strong $N-V_{\text {r }}$ transitions into the valencelike $1 \pi_{c}$ or $\pi^{*}$ orbital are also expected on this basis, since this shell is partially open. In addition to the presence of these predominantly valencelike $\sigma^{*}$ and $\pi^{*}$ orbitals, previous experience suggests the appearance of certain partially valence or pre-Rydberg $\delta_{z} 3 d, \delta_{u} 3 d, \pi_{u} 3 p$, and $\pi_{u} 3 d$ orbitals, ${ }^{48}$ and appropriate discrete and continuum Rydberglike orbitals, in the molecular oxygen excitation/ ionization spectra. ${ }^{1-6}$

Although the foregoing qualitative concepts are suggestive, the quantitative details of photoexcitation and ionization cross sections must, of course, be determined by appropriate calculations. In particular, the contributions of strong intravalence transitions to the discrete or continuous spectra are expected to be quite sensitive to the details of the calculations and, consequently, relatively large basis sets are required to obtain the correct separated-channel static-exchange results. Previous experience indicates that the valencelike orbitals indicated above can contribute substantially to the continuous spectra in first row diatomics. ${ }^{1-6}$

\section{A. $1 \pi_{g}$ spectra (I.P. $\left.=12.1 \mathrm{eV}\right)$}

In Table III and Fig. 1 are shown the results of the present calculations for $1 \pi_{\text {e }}$ excitation and ionization, 
TABLE IV. $1 \pi_{\mathrm{u}}$ excitation spectra in $\mathrm{O}_{2}($ I. P. $=16.1 \mathrm{eV})$

\begin{tabular}{|c|c|c|}
\hline $\begin{array}{l}\text { Present results }{ }^{\mathrm{a}} \\
\text { Energy }(\mathrm{eV}) / f \text { Number }\end{array}$ & $\begin{array}{l}\text { Experimental assignments } \\
\text { Energy }(\mathrm{eV}) / \text { Intensity }\end{array}$ & $\begin{array}{l}\text { Quantum-defect estimates } \\
\text { Energy }(\mathrm{eV}) / f \text { number }\end{array}$ \\
\hline \multicolumn{3}{|c|}{$\left(1 \pi_{\mathbf{u}}\right)^{3} \Sigma_{\boldsymbol{g}} \rightarrow\left(n s \sigma_{\boldsymbol{g}}\right)^{3} \Pi_{\mathfrak{u}}$} \\
\hline $12.59 / 0.0925$ & $12.35 /$ strong & $12.70 / 0.1361$ \\
\hline $14.70 / 0.0314$ & 14.53 & $14.59 / 0.0403$ \\
\hline $15.42 / 0.0141$ & 15.23 & $15.25 / 0.0170$ \\
\hline $15.76 / 0.0074$ & $\cdots$ & $15.56 / 0.0087$ \\
\hline $15.93 / 0.0044$ & $\cdots$ & $15.71 / 0.0050$ \\
\hline $16.03 / 0.0040$ & $\cdots$ & $15.82 / 0.0032$ \\
\hline \multicolumn{3}{|c|}{$\left(1 \pi_{u}\right)^{3} \Sigma_{\boldsymbol{g}}^{-}-\left(n d \pi_{g}\right)^{3} \Sigma_{u}^{-}$} \\
\hline $14.88 / 0.00097$ & $14.48 /$ medium & $14.48 / 0.00279$ \\
\hline $15.94 / \cdots$ & $\cdots$ & $15.21 / 0.00115$ \\
\hline \multicolumn{3}{|c|}{$\left(1 \pi_{u}\right)^{3} \Sigma_{g}^{-} \rightarrow\left(n d \delta_{g}\right)^{3} \Pi_{u}$} \\
\hline $14.80 / 0.0196$ & $14.58 /$ medium & $14.48 / 0,0669$ \\
\hline $15.53 / 0.0165$ & $\cdots$ & $15.21 / 0.0275$ \\
\hline
\end{tabular}

${ }^{\mathrm{a} A s}$ in Table III. The calculated energies are made to converge on the $a^{4} \Pi_{u} \mathrm{O}_{2}^{+}$ionic state, and the strengths refer to contributions from the series coverging on this state only, as is discussed further in the text.

balues taken from the tabulations of E. Lindholm, Ark. Fys. 40, 117 (1969). The entries refer to Rydberg series converging on the $a^{4} \Pi_{u}$ parent $\mathrm{O}_{2}^{+}$ionic state. Additional series converging on the $A^{2} \Pi_{u}$ ionic state are also reported at energies generally within $\sim 0.5 \mathrm{eV}$ of the values cited, although the series converging on the higher-lying $2^{2} \Pi_{u}(24 \mathrm{eV})$ and $5^{2} \Pi_{u}$ $(33 \mathrm{eV})$ ionic states also formed upon removal of a $1 \pi_{u}$ electron are apparently not reported As indicated in the text, the familiar $X^{3} \Sigma_{\xi}^{-} \rightarrow B^{3} \Sigma_{u}^{-}$Schumann-Runge transition is not included in the present calculations.

dValues obtai ned from the Rydberg formula and the Coulomb limit (Ref. 35) for $n=3,4, \ldots$ using quantum defects of 1.0 and 0.1 for the $n s$ and $n d$ series, respectively.

respectively. Referring to Table II, it is seen that $23 \sigma_{u}, 16 \pi_{u}$, and $9 \delta_{u}$ pseudospectra are used in calculating $1 \pi_{8}$ excitation and ionization cross sections. As indicated in the preceding section, the various Rydberg series are made to converge on the experimentally observed $X^{2} \Pi_{g} \mathrm{O}_{2}^{+}$adiabatic ionization potential. ${ }^{36-38}$ The predicted $1 \pi_{\varepsilon}-n \pi_{u}$ excitation series is evidently in good agreement with the positions and intensities of the ob-

TABLE V. $3 \sigma_{g}$ excitation spectra in $\mathrm{O}_{2}($ I.P. $=18.2 \mathrm{eV})$.

\begin{tabular}{|c|c|c|}
\hline $\begin{array}{l}\text { Present results }{ }^{2} \\
\text { Energy }(\mathrm{eV}) / f \text { Number }\end{array}$ & $\begin{array}{l}\text { Experimental assignments }{ }^{b} \\
\text { Energy (eV)/Intensity }\end{array}$ & $\begin{array}{l}\text { Quantum-defect estimates } \\
\text { Energy }(\mathrm{eV}) / f \text { Number }\end{array}$ \\
\hline \multicolumn{3}{|c|}{$\left(3 \sigma_{z}\right)^{3} \Sigma_{z}^{-} \rightarrow\left(n p \sigma_{u}\right)^{3} \Sigma_{u}^{-}$} \\
\hline $15.45 / 0.0825$ & $15.28 /$ medium & $15.63 / 0.1038$ \\
\hline $16.91 / 0.0307$ & 16.93 & $16.95 / 0.0352$ \\
\hline $17.45 / 0.0147$ & 17.44 & $17.46 / 0.0159$ \\
\hline $17.71 / 0.0081$ & 17.69 & $17.72 / 0.0085$ \\
\hline $17.85 / 0.0049$ & 17.83 & $17.86 / 0.0051$ \\
\hline $17.96 / \cdots$ & 17.91 & $17.94 / 0.0033$ \\
\hline \multicolumn{3}{|c|}{$\left(3 \sigma_{g}\right)^{3} \Sigma_{g}^{-} \rightarrow\left(n p \pi_{u}\right)^{3} \Pi_{u}$} \\
\hline $15.61 / 0.00622$ & $15.55 /$ strong & $15.63 / 0.00631$ \\
\hline $16.96 / 0.00206$ & 16.93 & $16.95 / 0.00214$ \\
\hline $17.47 / 0.00092$ & 17.45 & $17.46 / 0.00097$ \\
\hline $17.72 / 0.00049$ & $\cdots$ & $17.72 / 0.00052$ \\
\hline $17.90 / \cdots$ & $\cdots$ & $17.86 / 0.00031$ \\
\hline
\end{tabular}

${ }^{2} \mathrm{~A} s$ in Table III. The calculated energies are made to converge on the $b^{4} \Sigma_{z}^{-}$parent $\mathrm{O}_{2}^{+}$ionic state, and the strengths refer to contributions from series converging on this state only, as is discussed further in the text.

Spectral assignments taken from tabulations of J. Leclercq, Ann. Astrophys. 30, 93 (1967). See also the references cited in Footnote b of Table III. The entries refer to Rydberg series converging on the parent $\mathrm{O}_{2}^{+}$ionic $b^{4} \Sigma_{\xi}^{-}$state. Additional series converging on the ${ }^{2} \Delta_{k}$ and $B^{2} \Sigma_{\text {f }}^{-}$ionic states are also reported at energies generally within $\sim 2 \mathrm{eV}$ of the values cited. ${ }^{\mathrm{C}} \mathrm{As}$ in Table IV, employing $\delta=0.7$ for $n p$ series. 


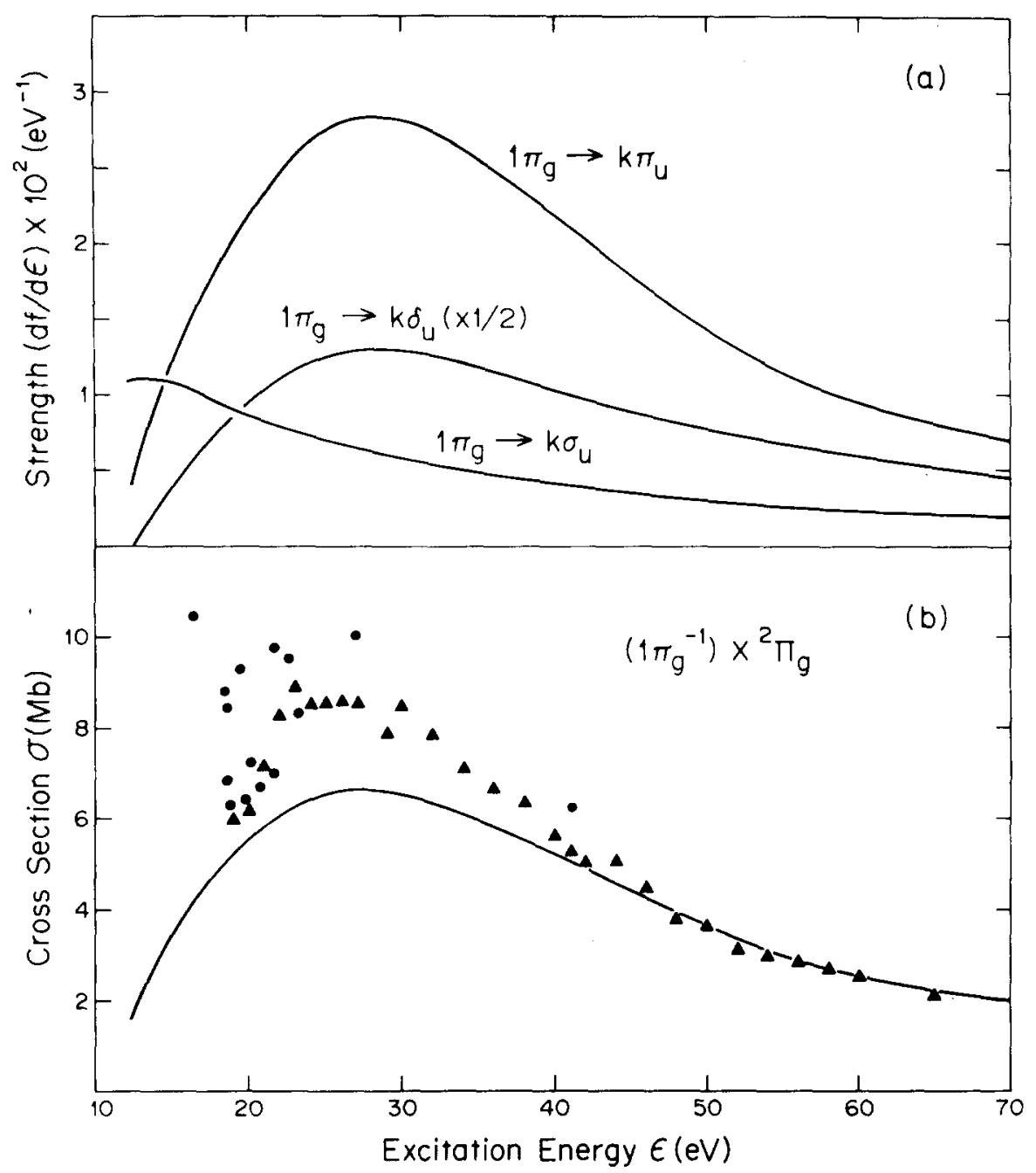

FIG. 1. (a) Vertical electronic $\mathrm{S}-\mathrm{T}$ oscillator-strength densities obtained from Eqs. (6) and appropriate multiplet factors for $1 \pi_{g} \rightarrow k \sigma_{u}, k \pi_{u}$, and $k \delta_{u}$ ionization in $\mathrm{O}_{2}$. (b) Partialchannel photoionization cross section for production of $\left(1 \pi_{g}^{-1}\right) X^{2} \Pi_{g} O_{2}^{+}$ions; $(\longrightarrow)$ vertical electronic S-T cross section; $\bullet$, line-source measurements (Ref. 25); $\wedge,(e, 2 e)$ coincidence measurements (Ref. 28). $1 \mathrm{Mb}=10^{-18} \mathrm{~cm}^{2}$. served $n p \pi_{u}$ series, ${ }^{21,49-51}$ and the first three members of the series are essentially identical with the earlier IVO calculations. ${ }^{50}$ The position of the $n=3$ member of this series, corresponding to excitation into a $3 p \pi_{u}$ Ryd berg orbital $\left(n^{*} \sim 2.2, \delta \sim 0.8\right)$, is taken from the recent electron impact-excitation measurements. ${ }^{50}$ The effective principle quantum number (2.2) of the resonance transition substantiates the $3 p \pi_{u}$ assignment, since Rydberg $p$-orbital quantum defects for first row diatomics are generally $\sim 0.6$ to $0.8 .^{51}$ of course the present cal culation neglects the Rydberg-valence interaction between the $\left(1 \pi_{c}^{-1} 3 p \pi_{u}\right)^{3} \Sigma_{u}^{-}$and $\left(1 \pi_{u}^{-1} 1 \pi_{q}\right)^{3} \Sigma_{u}^{-}$configurations, which apparently results in a shift of $\sim 0.4 \mathrm{eV}$ in the position of the first Rydberg state. ${ }^{52-54}$

In addition to the discrete Rydberg $n p \pi_{u}$ states in $\mathrm{O}_{2}$ indicated above, relatively compact pre-Rydberg atomic oxygen $3 p$ and $3 d$ orbitals (Table II) can also contribute to the $1 \pi_{g}-k \pi_{u}$ photoabsorption spectrum. ${ }^{48}$ Referring to $\mathrm{Fig.} \mathrm{1(a),} \mathrm{a} \mathrm{very} \mathrm{broad} \mathrm{and} \mathrm{strong} \mathrm{resonancelike}$ $\pi_{u} 3 d$ feature is evidently present in the $1 \pi_{q} \rightarrow k \pi_{u}$ photoionization cross section, having a maximum at $\sim 28 \mathrm{eV}$. An oxygen $d$-orbital interpretation of this feature is consistent with earlier theoretical studies, which indicate that compact $\pi_{u} 3 d$ oxygen orbitals do not contribute significantly to the discrete $1 \pi_{z} \rightarrow n \pi_{u}$ excitations in molecu lar oxygen. ${ }^{50}$ However, since a very large Gaussian basis set is employed in the present calculation, it is not possible to unambiguously assign the qualitative nature of this resonancelike feature by inspection of the appropriate IVO orbital vectors. Single-center expansions or computer plots of the resonancelike orbitals, which are not presented here, would provide useful information and are the subject of subsequent investigations. Moreover, diabatic calculation on configurational states of the type $\left(1 \pi_{\varepsilon}^{-1} \pi_{u} 3 d\right)^{3} \Sigma_{u}^{-}$and $\left(1 \pi_{g}^{-1} \pi_{u} 3 p\right)^{3} \Sigma_{u}^{-}$would also be helpful in this connection.

The $1 \pi_{u} \rightarrow n \sigma_{u}$ excitation series shown in Table III is evidently in good agreement with the experimental assignments and previous IVO calculations. ${ }^{49,50}$ Since the low-lying state at $\sim 8.5 \mathrm{eV}$ has such a small $f$ number it is not observed experimentally. The nature of this state, and of the entire $1 \pi_{q} \rightarrow n \sigma_{u} / k \sigma_{u}$ spectrum, is clarified by inspection of the appropriate potential curves, ${ }^{54}$ which indicate a diabatic $\left(1 \pi_{q}^{-1} \sigma_{u} 2 p\right)^{3} \Pi_{u}$ state crosses the $\left(1 \pi_{\varepsilon}^{-1} n p \sigma_{u}\right)^{3} \Pi_{u}$ Rydberg series almost vertically in the neighborhood of the $X^{3} \Sigma_{;}^{*}$ equilibrium internuclear separation. Rydberg-valence interaction results in the weak low-lying transition at $\sim 8.5 \mathrm{eV}$, with the diabatic intensity spreading over the higher Rydberg states and into the photoionization continuum. Indeed, there is evidence of a weak resonancelike $1 \pi_{s}-\sigma_{u} 2 p$ contribution just above threshold in the $1 \pi_{q} \rightarrow k \sigma_{u}$ photoionization cross section of 


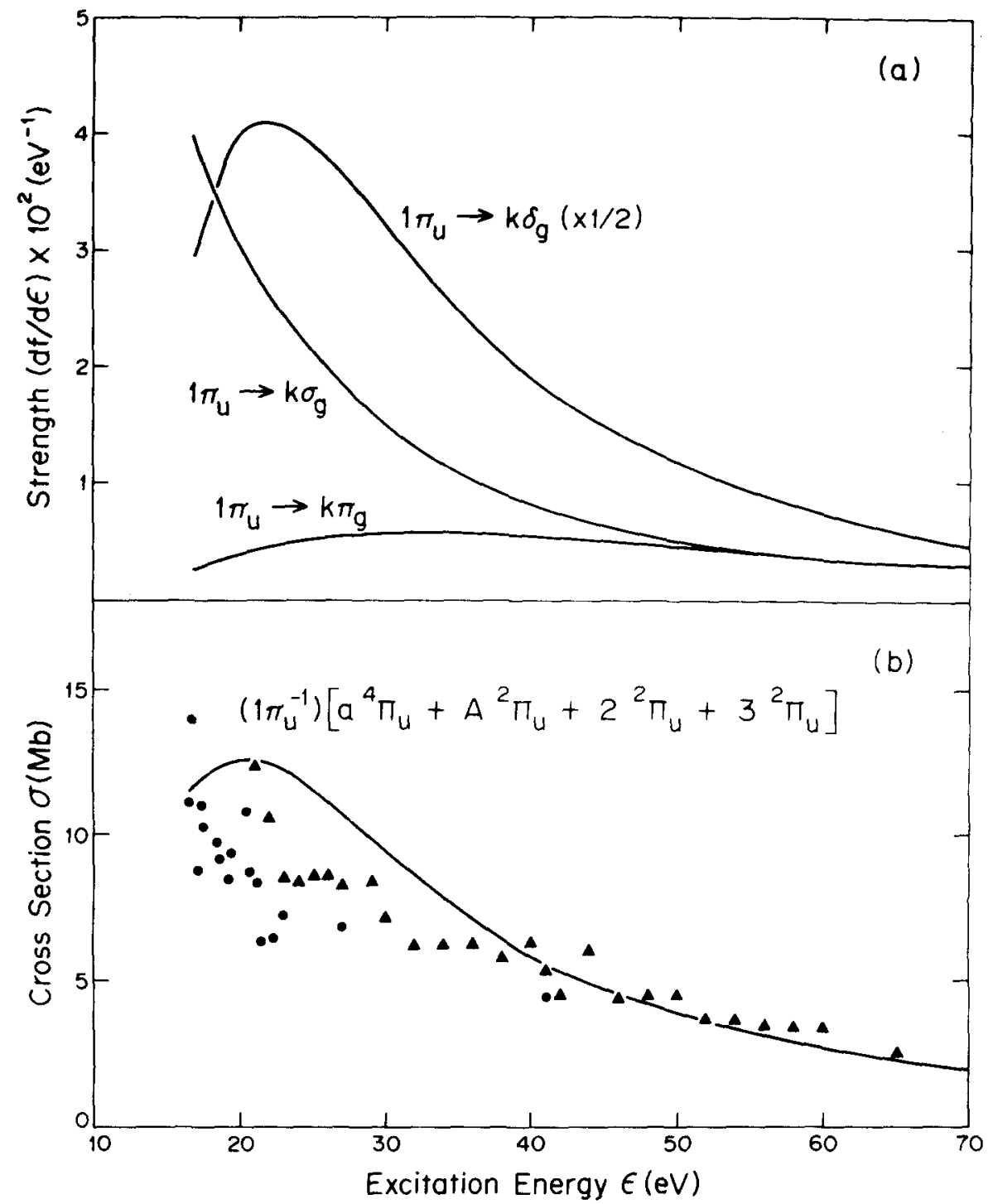

FIG. 2. (a) As in Fig. 1(a) for $1 \pi_{u}-k \sigma_{g}, k \pi_{g}$, and $k \delta_{g}$ photoionization. (b) As in Fig. 1(b) for the production of $a^{4} \Pi_{u}$, $A^{2} \Pi_{u}, 2^{2} \Pi_{u}$, and $3^{2} \Pi_{u} \mathrm{O}_{2}^{+}$ions. The $(e, 2 e)$ results are constructed as discussed in the text.
Fig. 1(a). This interpretation is also consistent with recent valence-basis CI calculations in $\mathrm{O}_{2}$, which locate the $1^{3} \Pi_{u}$ state at $\sim 11 \mathrm{eV},{ }^{55}$ although inspection of the appropriate vector is required to confirm this assignment. Interaction of the diabatic valence state with diffuse basis functions, not included in the CI calculations, ${ }^{55}$ presumably gives rise to the small shift to higher energy obtained from the present study. The second state in the $1 \pi_{p} \rightarrow n p \sigma_{u}$ series is perturbed but clearly Rydberg in nature with principle quantum $n=3$ and quantum defect $\delta \cong 0.5$. Evidently, the calculated $f$ numbers for the series are not entirely in accord with the approximate experimental intensity designation, a discrepancy that can be attributed to the sensitivity of transition strengths to Rydberg-valence interactions.

The $1 \pi_{e}-n \delta_{u}$ series of Table II is evidently weak, accounting for the lack of an experimental assignment in this case. Those values listed under "previous calcula tion" in Table III in this case are obtained from the Rydberg formula for $n=3$ and 4 employing a quantum defect of $0.1,{ }^{51}$ as is indicated in the table. The corresponding effective principle quantum numbers of 2.9 and 3.9 are in poor accord with the calculated IVO values of
3.55 and 4.93 , suggesting that a compact $\delta_{u} 3 d$ orbital contributes only weakly to the discrete $1 \pi_{\varepsilon} \rightarrow n \delta_{u}$ excitation series. However, referring to Fig. 1(a), a $3 d$ preRydberg atomic oxygen orbital can be regarded as contributing strongly to the $1 \pi_{s}-k \delta_{u}$ photoionization continuum at $\sim 28 \mathrm{eV}$, since Table II indicates that the $k \delta_{u}$ finalstate resonance feature can only be due to atomic oxygen $d$ orbitals. It is of particular interest to note that the peak in the cross section in this case is identical with that in the $1 \pi_{q} \rightarrow k \pi_{u}$ profile, substantiating in some measure the $3 d$ atomic oxygen character of both features (Table II). Diabatic calculations on the $\left(1 \pi_{g}^{-1} \delta_{u} 3 d\right)^{3} \Pi_{u}$ configurational state would be helpful in this connection.

Since the potential energy curve of the $X^{2} \Pi_{8} \mathrm{O}_{2}^{+}$state is deep and situated approximately vertically above the $X^{3} \Sigma \mathrm{O}_{2}$ ground state, ${ }^{40}$ the Franck-Condon factors favor only a few low-lying vibrational states, ${ }^{38}$ and the sum of the three curves of Fig. 1(a), which incorporate appropriate line-strength factors, ${ }^{56}$ provides a vertical-electronic approximation to the vibrationally averaged result that can be compared directly with corresponding crosssectional measurements. ${ }^{25-28}$ In Fig. 1(b) are shown the partial-channel IVO photoionization cross section for 


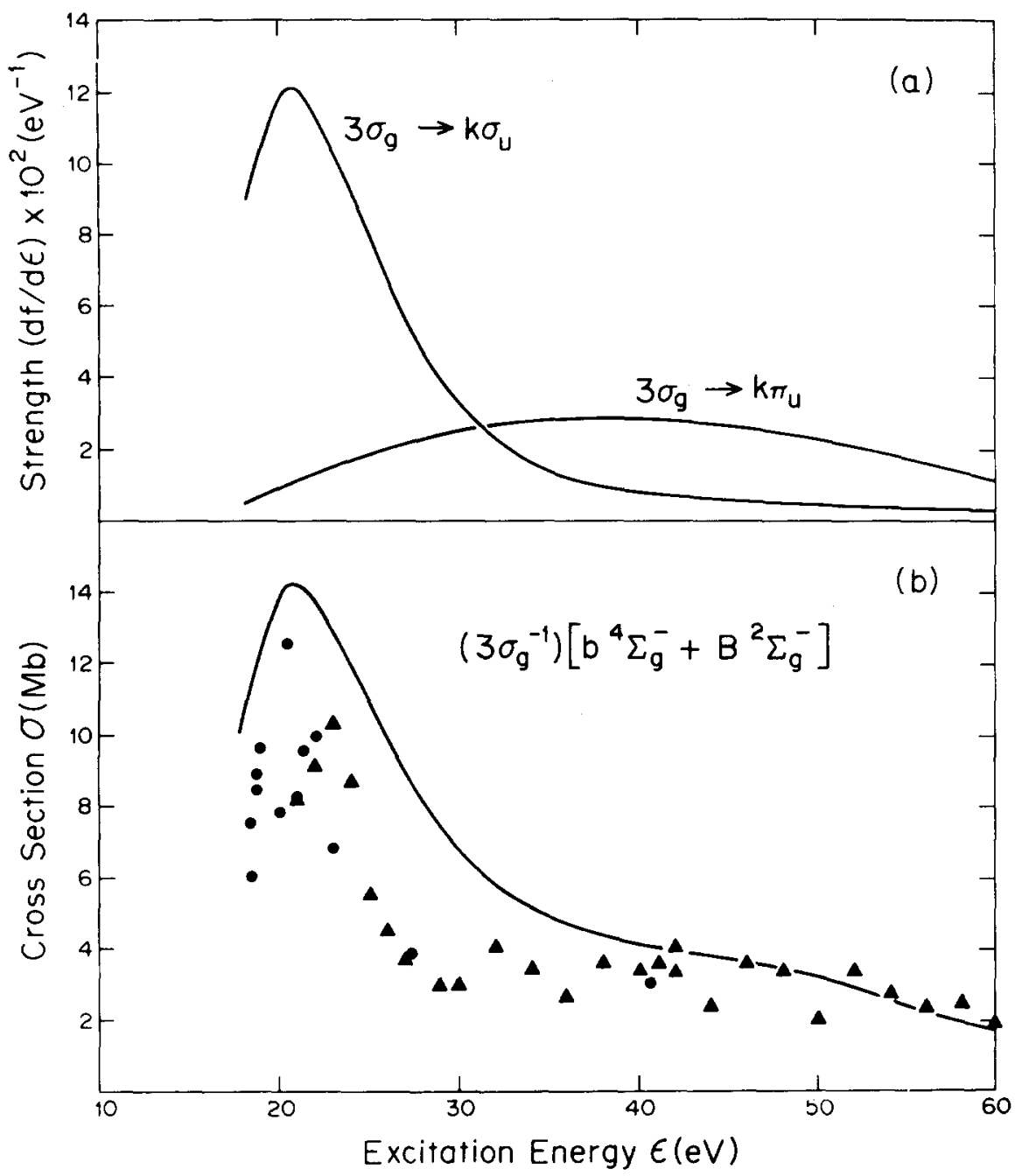

FIG. 3. (a) As in Fig. 1(a) for $3 \sigma_{g}-k \sigma_{u}$ and $k \pi_{u}$ photoionization. (b) As in Fig. 1(b) for the production of $b^{4} \Sigma_{g}^{-}$and $B^{2} \Sigma_{g}^{-} \mathrm{O}_{2}^{+}$ions. The $(e, 2 e)$ results are constructed as discussed in the text.

production of $X^{2} \Pi_{,} \mathrm{O}_{2}^{+}$parent ions and corresponding results obtained from recent line-source ${ }^{25}$ and $(e, 2 e) \mathrm{di}-$ pole oscillator-strength measurements. ${ }^{28}$ Although there is considerable structure in the line-source measurements at lower energy, which is presumably due to the presence of autoionizing Rydberg series converging on higher ionic states and to vibrational structures, ${ }^{40}$ and there is a small quantiative discrepancy at intermediate energy, it is quite clear that the experimental and theoretical values are in good agreement. The small quantitative discrepancy near the maximum at $\sim 28 \mathrm{eV}$ in Fig. 1(b) can perhaps be attributed to the relatively small $9 \delta_{u}$ basis employed (Table II), presumably making the description of the $1 \pi_{s} \rightarrow k \delta_{u} 3 d$ resonance feature in Fig. 1(a) somewhat imprecise. It is particularly satisfying that the calculations for this channel are in good agreement with experiment at higher energies, where the resonance feature does not contribute and a reliable description of the scattering functions can be expected in the basis employed. The experimental data of Fig. 1(b) evidently provides substantial support for the presence of of the $1 \pi_{g}-k \pi_{u} 3 d$ and $k \delta_{u} 3 d$ resonances in the $\left(1 \pi_{q}^{-1}\right) X^{2} \Pi_{g}$ cross-section components of Fig. 1(a).

\section{B. $1 \pi_{u}$ spectra (I.P. $\left.=16.1 \mathrm{eV}\right)$}

The calculated $1 \pi_{u}$ excitation series are compared with available spectral assignments and quantum-defect esti- mates in Table IV. Referring to Table II, it is seen that $23 \sigma_{\varepsilon}, 16 \pi_{\epsilon}$, and $13 \delta_{\text {, }}$ pseudospectra are employed in this case. The indicated adiabatic ionization potential refers to the $a^{4} \Pi_{u}$ ionic state, and consequently, the calculated excitation series are made to converge on this value [Eq. (6a)]. Moreover, the calculated strengths cited refer only to the $a^{4} \Pi_{u}$ contributions, which correspond to $\frac{2}{3}$ of the entire transition array. ${ }^{56}$ Corresponding experimentally assigned series converging on the $A^{2} \Pi_{u}$ ionic state are generally within $\sim 0.5 \mathrm{eV}$ of the values cited, ${ }^{51}$ although the higher-lying series converging on the $2^{2} \Pi_{4}$ and $3^{2} \Pi_{u} \mathrm{O}_{2}^{+}$states have apparently not been studied. The $2{ }^{2} \Pi_{u}$ state in $\mathrm{O}_{2}^{+}$is reported at $\sim 24 \mathrm{eV}$, and the unassignsigned band at $\sim 33 \mathrm{eV}$ in the photoelectron spectra is attributed he re to the $3^{2} \Pi_{u}$ state. ${ }^{28}$ As indicated in the preceding section, the static-exchange approach employed here predicts the appearance of four parent $\mathrm{O}_{2}^{+}$ ionic state $\left[a^{4} \Pi_{u}, A^{2} \Pi_{u}, 2^{2} \Pi_{u}, 3^{2} \Pi_{u}\right]$, and corresponding partial-channel photoionization cross sections, upon removal of a $1 \pi_{\mathrm{w}}$ electron. Since the discrete states of Table IV lie above the $12.1 \mathrm{eV} 1 \pi$, ionization potential, they can autoionize into the underlying continua. Although autoionization linewidth calculations are not reported here, the calculated positions should be relatively insensitive to the appropriate interaction with the background continua.

The calculated $1 \pi_{u} \rightarrow n \sigma_{g}$ series is evidently in general 


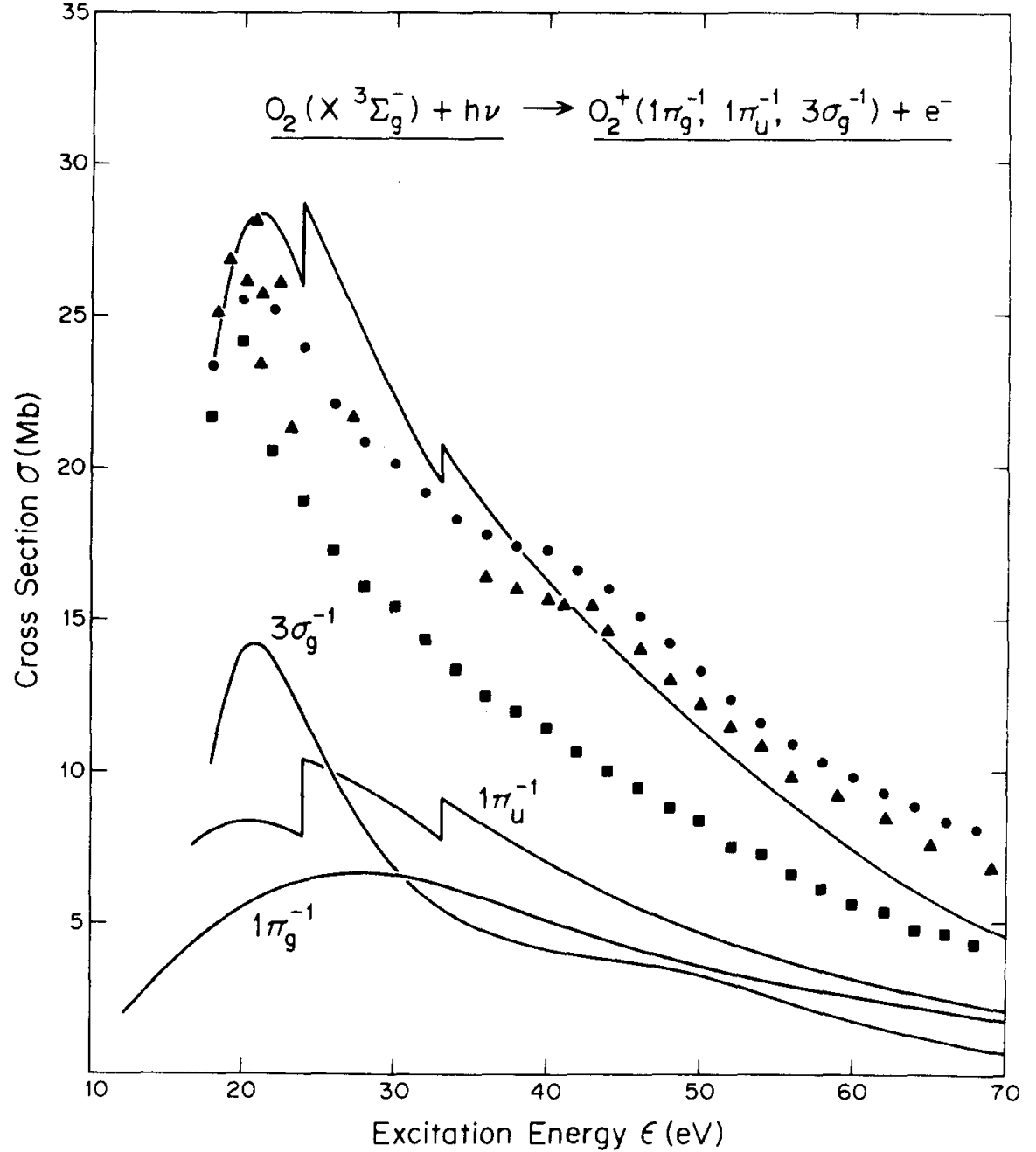

FIG. 4. Valence-shell photoabsorption and ionization cross sections in $\mathrm{O}_{2}$. S-T results as indicated; Experimental results; •, total absorption cross section from electronimpact dipole oscillatorstrength studies (Ref. 28); a photoabsorption measurements of the total cross section (Refs. 25 and 26); m, $\mathrm{O}_{2}^{+}$photoionization cross section from $(e, e+i o n)$ dipole oscillator-strength studies (Ref. 28). $1 \mathrm{Mb}$ $=10^{-18} \mathrm{~cm}^{2}$. agreement with the assigned $n s \sigma_{q}$ series leading to the $a^{4} \Pi_{u}$ ionic state, and when shifted by $\sim 0.5 \mathrm{eV}$ with the corresponding series at slightly higher energy converging on the $A^{2} \Pi_{u}$ ionic state (not shown). ${ }^{51}$ Moreover, both IVO and experimental values are in general agreement with the positions obtained from the Rydberg formula and appropriate quantum defects, also listed in the table. The $n=3$ resonance in the series is evidently $3 s \sigma_{s}$ Rydberg in character $\left(n^{*} \sim 2, \delta \sim 1\right)$, and there is no evidence of substantial valence-orbital contribution to this particular subchannel, since the corresponding photoionization profile [Fig. 2(a)] is monotonically decreasing. These results are in general accord with expectations and with previously reported studies of the $1 \pi_{u}$ $\rightarrow n \sigma_{\ell} / k \sigma_{\varepsilon}$ and $1 \pi-n \sigma / k \sigma$ cross sections in $\mathrm{N}_{2}$ and $\mathrm{CO}$, respectively. ${ }^{2,3}$

There is no evidence of a strong $1 \pi_{u}-1 \pi_{l}$ transition in the present calculations due to the orthogonality constraint imposed, as indicated above. Since this excitation corresponds to the familiar $X^{3} \Sigma_{z}^{-}-B^{3} \Sigma_{u}^{-}$SchumannRunge system, ${ }^{52,53}$ it is not necessary to perform a separate investigation of its position and intensity here. The experimentally assigned first member of the $n d \pi_{r}$ series is in some accord with the present calculations, although the theoretical position is higher, perhaps as a consequence of the orthogonality constraint, and the predicted intensity is apparently weak. The IVO $1 \pi_{u}$ $\rightarrow n \pi_{g}$ calculations are in reasonable agreement with the positions obtained from the Rydberg formula and appropriate quantum defects, ${ }^{51}$ also show $\mathrm{n}$ in the table. Referring to Fig. 2(a), the corresponding $1 \pi_{u}-k \pi_{g}$ photoionization cross section is quite weak, although there is evidence of a very broad resonancelike feature at $\sim 30 \mathrm{eV}$. Apparently the $1 \pi_{u}^{-1}$ effective potential supports only one valencelike $\pi_{\boldsymbol{c}}$ orbital, which appears in the discrete spectrum in this case (Schumann-Runge transition). The $1 \pi_{u}-k \pi_{2}$ cross section in $\mathrm{O}_{2}$ of $\mathrm{Fig}, 2(\mathrm{a})$ is apparently dissimilar in appearance from the previously reported corresponding $1 \pi_{u} \rightarrow k \pi_{z}$ and $1 \pi \rightarrow k \pi$ cross sections in $\mathrm{N}_{2}$ and $\mathrm{CO}$, respectively, which exhibit large peaks just above threshold. ${ }^{2,3}$ Reexamination of the previously reported static-exchange calculations of $1 \pi_{u}$ and $1 \pi$ excitation and ionization spectra in $\mathrm{N}_{2}$ and $\mathrm{CO}$, however, indicates that $1 \pi_{\mu} \rightarrow k \pi_{\varepsilon}$ and $1 \pi-k \pi$ photoionization cross sections similar to that of Fig. 2(a) are obtained when appropriate projection techniques are used to isolate the strong $\pi \rightarrow \pi^{*}$ transitions in each case. 57

The experimentally assigned first member of the ${ }^{1} \pi_{u}$ $\rightarrow n d \delta$, series is in some accord with the present calculations, although the IVO resonance transition apparently contains only weak contributions from $3 d$ orbitals, since 
$n^{*}=3.2$ in this case. Indeed, much of the intensity in this IVO channel is concentrated in the strong resonance

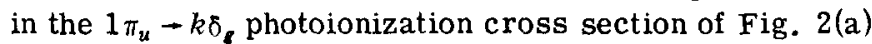
at $\sim 22 \mathrm{eV}$. This feature can be attributed to the presence of compact $\delta_{z} 3 d$ molecular orbitals in final state symmetry, similar to the case of the $1 \pi_{g}-k \delta_{u}$ channel [Fig. 1(a)). Moreover, the $1 \pi_{u}-k \delta_{z}$ profile of Fig. 2(a) is highly similar to the previously reported corresponding $1 \pi_{u} \rightarrow k \delta_{\text {, }}$ and $1 \pi \rightarrow k \delta$ photoionization cross sections in $\mathrm{N}_{2}$ and $\mathrm{CO}$, respectively. ${ }^{2,3} \mathrm{As}$ in these latter cases, diabatic calculations on the $\left(1 \pi_{u}^{-1} \delta_{z} 3 d\right)^{3} \Pi_{u}$ state in $\mathrm{O}_{2}$ would be helpful in clarifying the nature of the resonancelike feature in this channel.

The $a^{4} \Pi_{u}$ and $A^{2} \Pi_{u} \mathrm{O}_{2}^{+}$ionic state potential curves are not as deep as is that of the $X^{2} \Pi_{f}$ ionic state, and their minima are shifted to somewhat larger internuclear separations $\left(R_{e} \sim 2.8 a_{0}\right) 0^{40}$ Moreover, the $2^{2} \Pi_{u}$ and $3^{2} \Pi_{u}$ state curves are most likely repulsive. ${ }^{40}$ Nevertheless, the Franck-Condon regions are still expected to be relatively limited in these cases, ${ }^{38}$ and the sum of the three vertical photoionization curves of Fig. 2(a) should provide a vibrationally averaged cross section suitable for comparison with appropriately interpreted measured data. In Fig. 2(b) the theoretically determined vertical electronic cross section so obtained for formation of $a^{4} \Pi_{u}, A^{2} \Pi_{u}, 2^{2} \Pi_{u}$, and $3^{2} \Pi_{u}$ parent $\mathrm{O}_{2}^{+}$ionic states is compared with the results of recent line-source ${ }^{25}$ and $(e, 2 e)^{28}$ dipole oscillator-strength measurements. The $a^{4} \Pi_{u}$ and $A^{2} \Pi_{u}$ contributions are obtained from the $(e, 2 e)$ partial-channel data by subtracting the $b^{4} \Sigma_{g}^{-}$channel contribution from the measured values corresponding to the sum of the $a^{4} \Pi_{u}, A^{2} \Pi_{u}$, and $b^{4} \Sigma_{\text {, channels. }}{ }^{28}$ Since the $b^{4} \Sigma_{z}^{-}$cross section is not resolved by the $(e, 2 e)$ measurements, an approximation to its contribution is obtained using twice the measured $B^{2} \Sigma_{g}^{-}$cross section (which is experimentally resolved), ${ }^{28}$ employing the theoretically predicted ratio $b^{4} \Sigma_{-}^{-} / B^{2} \Sigma_{s}^{-}=2{ }^{56}$ Support for this theoretically determined ratio is provided by the line-source branching-ratio measurements of the $b^{4} \Sigma_{g}^{-}$and $B^{2} \Sigma_{z}^{-}$contributions, ${ }^{25}$ which are precisely in the ratio $2: 1$ at the highest energy reported. To the sum of $a^{4} \Pi_{u}$ and $A^{2} \Pi_{u}$ partial-channel cross sections are added the $2^{2} \Pi_{u}$ contribution and the separately resolved contribution appearing at $33 \mathrm{eV}$ in the photoelec tron spectra, ${ }^{28}$ which is here assigned as the $3^{2} \Pi_{u} \mathrm{O}_{2}^{+}$ parent ionic state. The $2^{2} \Pi_{u}$ cross section is separated from the $c^{4} \Sigma_{u}^{*}$ channel with which it is degenerate by making use of the corresponding line-source data, ${ }^{25}$ which indicates the former contributes $\sim 70 \%$ to the combined $2{ }^{2} \Pi_{u}$ and $c^{4} \Sigma_{u}^{-}$bands. In adding the $2^{2} \Pi_{u}$ and $3^{2} \Pi_{u}$ contributions to the $a^{4} \Pi_{u}$ and $A^{2} \Pi_{u}$ cross sections it is, of course, necessary to refer the former to the $a^{4} \Pi_{u}$ threshold at $\sim 16 \mathrm{eV}$. Although this procedure perhaps takes some liberties with the experimental data, it is seen from Fig. 2(b) that generally good aggreement be tween theory and experiment is obtained. The structure in the measurements at lower energy is presumably a consequence of autoionizing states and vibrational features. Since the calculated $1 \pi_{u} \rightarrow k \delta_{z} 3 d$ resonance appears in this spectral region, the measurements do not provide an unambigous experimental verification in this case, although the experimental results are compatible with its presence. Moreover, the $2^{2} \Pi_{u}$ and $3^{2} \Pi_{u}$ partial-channel cross sections separately have maxima $\sim 5 \mathrm{eV}$ above their thresholds, in general support of the $1 \pi_{u} \rightarrow k \delta_{g} 3 d$ feature of Fig. 2(a).

\section{C. $3 \sigma_{g}$ spectra (I.P. $\left.=18.2 \mathrm{eV}\right)$}

The calculated $3 \sigma_{z}$ excitation series and ionization cross sections are shown in Table $\mathrm{V}$ and $\mathrm{Fig}$. 3, respectively. Table II indicates that $23 \sigma_{u}, 16 \pi_{u}$, and $9 \delta_{u}$ pseudospectra are employed in calculating the $3 \sigma_{g}$ excitation and ionization cross sections. The calculated energies are made to converge on the experimentally observed $b^{4} \Sigma_{g}^{-} \mathrm{O}_{2}^{+}$state, and the strengths shown correspond to contributions from series converging on this state only, which accounts for $\frac{2}{3}$ of the entire transition array. ${ }^{56}$ Evidently the $3 \sigma_{g} \rightarrow n \sigma_{u}$ IVO series is in very good agreement with the assigned $n p \sigma_{u}$ series, ${ }^{51,58}$ and with estimates obtained from quantum defects. Of course, the effects of autoionization must be included in order to obtain a quantitative comparison of intensities. The $n=3$ resonance transition in the series is essentially Rydberg $\left(n^{*} \sim 2.2, \delta \sim 0.8\right)$ in character, and there is no evidence of a strong $3 \sigma_{g} \rightarrow \sigma_{u} 2 p$ intravalence excitation present in the discrete spectrum. However, referring to Fig. 3(a), the $3 \sigma_{z}-k \sigma_{u}$ IVO photoionization channel has a prominent resonancelike feature at $\sim 20 \mathrm{eV}$, which can be attributed to the presence of valencelike $\sigma_{u} 2 p$ orbitals in the $k \sigma_{u}$ photoionization continuum. Similar resonances appear in the $k \sigma_{u}$ and $k \sigma$ continua of $\mathrm{N}_{2}$ and $\mathrm{CO}$, respectively, ${ }^{1-3}$ and presumably related valencelike diabatic $2^{3} \Sigma_{u}^{-}, 3^{3} \Sigma_{u}^{-}$states corresponding to $b^{4} \Sigma_{p}^{-}$and $B^{2} \Sigma_{p}^{-}$parentages, respectively, are obtained at 18.4 and $20.9 \mathrm{eV}$ from recent CI calculations. ${ }^{55}$

The calculated $3 \sigma_{g}-n \pi_{u}$ excitations are evidently in very good agreement with the experimental positions and quantum defect estimated for the $n p \pi_{u}$ series, although the strong intensity assignment is clearly at variance with the theoretical results. Evidently, the corresponding $3 \sigma_{\boldsymbol{p}} \rightarrow k \pi_{u}$ photoionization cross section [Fig. 3(a)] is very weak, although there is some evidence of a broad high-energy maximum at $\sim 30$ to $40 \mathrm{eV}$, presumably corresponding to the associated resonance at $\sim 30 \mathrm{eV}$ in the $1 \pi_{\boldsymbol{g}} \rightarrow k \pi_{u}$ cross section [Fig. 1(a)].

The Franck-Condon factors for production of $b^{4} \Sigma^{-}$

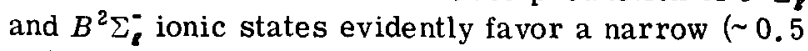
$\mathrm{eV}$ ) band of low-lying vibrational states, and do not extend into the dissociative photoionization continuum. ${ }^{38,40}$ Consequently, the two subchannel components for vertical photoionization of Fig. 3(a) are summed and compared in Fig. 3(b) with the results of recent line-source ${ }^{25}$ and $(e, 2 e)^{28}$ dipole oscillator-strength measurement. The $(e, 2 e)$ results shown are simply three times the measured $B^{2} \Sigma_{c}^{-}$channel cross section, making use of the theoretically predicted ratio $b^{4} \Sigma_{c}^{-} / B^{2} \Sigma_{-}^{-}=2$ indicated previously. ${ }^{56}$ Although the theoretical and experimental results are evidently in general agreement, it is not entirely clear if the predicted $3 \sigma_{g}-k \sigma_{u}$ resonance feature is experimentally verified, since it appears near threshold in the region of possible vibrational and autoionizing structure. Nevertheless, the presence of a resonancelike feature just above threshold in the $3 \sigma_{g} \rightarrow k \sigma_{u}$ cross 
TABLE VI. Photoionization resonance assignments in molecular oxygen.

\begin{tabular}{lcc}
\hline \hline $\begin{array}{l}\text { Valence-basis CI calculations }{ }^{2} \\
\text { State/Energy (eV) }\end{array}$ & $\begin{array}{l}\text { Static-exchange calculations }{ }^{b} \\
\text { Transition/Energy (eV) }\end{array}$ & $\begin{array}{c}\text { Experiment }^{\mathrm{c}} \\
\text { Energy (eV) }^{2}\end{array}$ \\
\hline & ${ }^{3} \Sigma_{u}^{-}$resonances \\
$B^{3} \Sigma_{u}^{-} / 9.03$ & $1 \pi_{u} \rightarrow 1 \pi_{g} 2 p / \cdots$ & $\sim 6$ \\
$2,3^{3} \Sigma_{u}^{-} / 18.4,20.9$ & $3 \sigma_{g} \rightarrow k \sigma_{u} 2 p / 21$ & $\sim 21$ \\
$\ldots$ & $1 \pi_{g} \rightarrow k \pi_{u} 3 d / 28$ & $\sim 26$ \\
& ${ }^{3} \Pi_{u}$ resonances & \\
$1{ }^{3} \Pi_{u} / 10.5$ & $1 \pi_{g} \rightarrow k \sigma_{u} 2 p / 12$ & $\cdots$ \\
$\ldots$ & $1 \pi_{u} \rightarrow k \delta_{g} 3 d / 22$ & $\sim 20$ \\
$\ldots$ & $1 \pi_{g} \rightarrow k \delta_{u} 3 d / 28$ & $\sim 26$ \\
\hline \hline
\end{tabular}

${ }^{2}$ R. P. Saxon and B. Liu, J. Chem. Phys. 67, 5432 (1977). The indicated values refer to vertical excitation energies at $R=2.3 a_{0}$. See also R. J. Buenker and S. D. Peyerimhoff, Chem. Phys. 8, 324 (1975); Chem. Phys. Lett. 34, 225 (1975).

balues obtained from Tables III to V and Figs. 1 to 4 .

${ }^{\mathrm{c}}$ Estimates obtained from the references cited and data appearing in Figs. 1 to 4 .

section is consistent with the experimental data of Fig. 3 (b), with the absence of a strong intravalence transition in the corresponding discrete spectrum (Table V), with the appearance of a similar weak resonancelike feature in the $1 \pi_{\varepsilon} \rightarrow k \sigma_{u}$ cross section of Fig. 1(a), and with the recent valence-basis calculations for the $2^{3} \Sigma_{u}^{-}$ and $3^{3} \Sigma_{u}^{-}$diabatic states. ${ }^{55}$ Moreover, it is of considerable interest to note that measurements of the $K$-edge photoabsorption cross section in $\mathrm{O}_{2}$ indicate the presence of a resonancelike feature at or just above the photoionization threshold. ${ }^{22-24}$ This feature presumably can be assigned to the $1 \sigma_{z} \rightarrow k \sigma_{u}$ channel, and corresponds to the related $3 \sigma_{q} \rightarrow k \sigma_{u}$ resonance of Fig. $3(\mathrm{a})$, providing further indirect support for the presence of a valencelike $\sigma_{u} 2 p$ contribution to the $k \sigma_{u}$ continuum in $\mathrm{O}_{2}$.

\section{Valence-shell photoabsorption cross section}

A further comparison between theory and experiment is provided by the results of recent synchrotron radiation, ${ }^{26}$ and electron-impact and $(e, e+$ ion) coincidence studies ${ }^{28}$ of total absorption and $\mathrm{O}_{2}^{+}$and $\mathrm{O}^{+}$photoproduc tion in $\mathrm{O}_{2}$ in the 18 to $70 \mathrm{eV}$ energy interval. In order to obtain a meaningful comparison between theory and experiment in this case it is necessary to refer the $2^{2} \Pi_{u}$ and $3^{2} \Pi_{u}$ partial -channel contributions to the appropriate thresholds (24 and $33 \mathrm{eV}$, respectively), rather than to the $a^{4} \Pi_{u}$ threshold, as in Fig. 2(b). When this procedure is followed, the $1 \pi_{u}^{-1}$ contribution will exhibit three distinct thresholds, associated with the $a^{4} \Pi_{u}$ and $A^{2} \Pi_{u}, 2^{2} \Pi_{u}$, and $3^{2} \Pi_{u}$ states, respectively. In the absence of appropriate line shape calculations, the contributions to the total cross section from autoionizing states converging on the $2^{3} \Pi_{u}$ and $3^{2} \Pi_{u}$ thresholds are neglected, resulting in small discontinuities in these cases. Alternatively, if the states below these two thresholds are included in the S-T procedure, corresponding to introduction of very broad autoionization widths, the discontinuities are no longer present and the cross section increases somewhat in these regions. Finally, the relative contributions of the $A^{2} \Pi_{u}, 2^{2} \Pi_{u}$, and $3{ }^{2} \Pi_{u}$ partial-channel cross sections are obtained from a simple three-term $\mathrm{O}_{2}^{+}$configuration-interaction calculation in which the experimentally observed thresholds are introduced as constraints. The results of this semiempirical analysis, which is in the spirit of the use of experimental ionization thresholds in Eqs. (6), indicate that the $A^{2} \Pi_{u}$ cross section is very weak, whereas the $2^{2} \Pi_{u}$ and $3^{2} \Pi_{u}$ contributions are in the ratio $\sim 2: 1$. These predictions are in accord with the measured $(e$, 2e) $2^{2} \Pi_{u}$ and $3^{2} \Pi_{u}$ cross sections, ${ }^{28}$ and with the apparent weak contribution the $A^{2} \Pi_{u}$ state makes to the highresolution photoelectron spectra, ${ }^{38}$ although not with previously reported theoretical studies. ${ }^{59}$

In Fig. 4 the results of the present IVO calculations for photoionization of the $1 \pi_{\varepsilon}, 1 \pi_{u}$, and $3 \sigma_{\varepsilon}$ orbitals in $\mathrm{O}_{2}$ constructed as indicated above, are compared with measured cross sectional values. Evidently, the theoretical results are in good agreement with the measured photoabsorption cross section at energies $\leq 30 \mathrm{eV}$, whereas the measured photoabsorption cross section is greater than the theoretical result above $\sim 30 \mathrm{eV}$, presumably as a consequence of dissociative $\left(2 \sigma_{g}, 2 \sigma_{u}\right)$ and two-electron photoionization. Moreover, the inflection in the data at $\sim 40 \mathrm{eV}$ can be attributed to an increase in $\mathrm{O}^{+}$production at this energy. ${ }^{28}$ At higher energies the calculations approach the measured photoionization cross section for $\mathrm{O}_{2}^{+}$production, and consequently are in ac cord with expectations. Although the total cross sectional measurements do not provide direct evidence regarding resonance features, the presence of a maximum just above threshold in the measured values is not incompatible with the calculations, providing additional indirect support for the $3 \sigma_{z} \rightarrow k \sigma_{u}$ resonance.

\section{RESONANCE FEATURES}

The various resonance features appearing in the partial-channel photoabsorption cross sections in molecular oxygen warrant further summarizing discussion. Table VI provides a summary of the theoretical assignments and positions, and of the corresponding 
experimental estimates. The energies given in the first column are taken from recent valence-basis configuration-interaction calculations, ${ }^{55}$ which refer to diabatic states, rather than spectroscopic states. Evidently, the predicted IVO and CI positions are in general agreement for the lowest-lying states of each symmetry. This suggests, as indicated above and discussed further below, that the positions of resonances in photoionization cross sections are determined in large measure by the diabatic approximation, although the shapes of the resonance cross sections are sensitive to the details of Rydberg-valence interaction in the continuum. Table VI emphasizes the very good agreement obtained be tween the separated-channel static-exchange results and the estimated positions of the measured photoionization resonances.

Although the results of $\mathrm{T}$ able VI are satisfactory, it is desirable to provide a somewhat more detailed characterization of the resonance features in the photoionization profiles in molecular oxygen. Referring to Fig. 1(a), the $1 \pi_{g}-k \pi_{u}$ and $1 \pi_{q}-k \delta_{u}$ resonances are at tributed in the foregoing discussion (Sec. IV.A) to contributions to the photoionization continua from relatively compact pre-Rydberg $3 d$ atomic oxygen orbitals (Table II). This view is in accord with the observation that $3 d$ oxygen orbitals do not contribute significantly to the discrete $1 \pi_{g} \rightarrow n \pi_{u}$ excitation series, ${ }^{50}$ and with the evident highly similar natures of the $1 \pi_{g} \rightarrow k \pi_{u}$ and $1 \pi_{\varepsilon} \rightarrow k \delta_{u}$ profiles. Nevertheless, it is only possible to make an unambiguous assignment in the case of the $1 \pi_{z} \rightarrow k \delta_{u}$ profile, since the supplemental basis functions employed are all of $d_{x y}$ type in this case (Table II). For the $1 \pi_{g}$ $\rightarrow k \pi_{u}$ profile it is possible that the relatively compact portion of the oxygen and CM $p_{x}$ basis (Table II) can contribute significantly to the resonance feature. Of course, the presence of a well-defined $n=3$ member in the discrete $n p \pi_{u}$ series (Table III) makes this possibility somewhat less likely. Configuration-interaction calculations that include the $\left(1 \pi_{g}^{-1} \delta_{u} 3 d\right)^{3} \Pi_{u}$ and $\left(1 \pi_{g}^{-1} \pi_{u} 3 d\right)^{3} \Sigma_{u}^{-}$ configurational states, which are apparently unavailable at present, would be helpful in this connection, as indicated above. Presumably the appropriate diabiatic curves, which should correlate with the dipole allowed $\left(2 p^{-1} 3 d\right)^{3} D^{0}$ atomic oxygen state in the separated-atom limit, cross the outer limbs of the Rydberg series con-

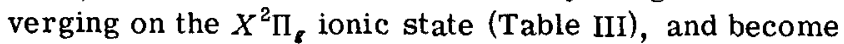
photoionization resonances at the ground-state equilibrium internuclear separation. By contrast, the $\left(1 \pi_{p}^{-1} \pi_{u} 3 p\right)^{3} \Sigma_{u}^{-}$ diabatic state presumably correlates with the $\left(2 p^{-1} 3 p\right)^{5} p^{e}$ atomic oxygen state in the separated-atom limit, and, consequently, is not dipole connected to the ground state in this limit. It is not possible to provide an unambigous molecular-orbital characterization of the small but distinct feature near threshold in the $1 \pi_{g} \rightarrow k \sigma_{u}$ channel [Fig. 1(a)] on the basis of the present calculations. How ever the absence of a strong valencelike member in the calculated and observed $n p \sigma_{u}$ excitation series (Table III), as well as the results of previously reported CI calculations, ${ }^{54,55}$ which indicate the presence of a diabatic valence state crossing the Rydberg series, suggests that this small feature can be attributed to a compact $\sigma_{u} 2 p$ orbital lying just above threshold. Indeed, referring to
Table VI, the position of the diabatic $\left(1 \pi_{\varepsilon}^{-1} \sigma_{u} 2 p\right)^{3} \Pi_{u}$ state is seen to be in rather good agreement with the present IVO result. Since only the $X^{2} \Pi_{g} \mathrm{O}_{2}^{+}$ionic state is produced upon removal of a $1 \pi_{\text {e }}$ electron, the calculated or bital photoionization cross sections [Fig. 1(a)] are simply related to the experimental results [Fig. 1(b)], which later provide considerable support for the existence of the predicted $\pi_{u} 3 d$ and $\delta_{u} 3 d$ resonance features.

Referring now to the $1 \pi_{u}$ photoionization profiles of Fig. 2(a), the prominent feature in the $1 \pi_{u}-k \delta_{s}$ subchannel can be attributed to contributions from relatively compact pre-Rydberg $3 d$ atomic oxygen orbitals (Table II), in accordance with the observation that the discrete resonance in the $n d \delta_{\text {, }}$ series (Table IV) has an effective principle quantum number of $\sim 3.2$. Evidently, the opening of the $1 \pi_{u}$ shell provides a somewhat more attractive potential for the $k \delta_{g}$ spectrum than does the $1 \pi_{g}$ shell for the $k \delta_{u}$ spectrum, since the resonance in the former appears at significantly lower energy $(\sim 5 \mathrm{eV}$ above threshold) than does the latter ( $16 \mathrm{eV}$ above threshold). Recall that a prominent $1 \pi_{u} \rightarrow k \delta_{\varepsilon}$ resonance feature also appears in the previously reported corresponding cross section in $\mathrm{N}_{2}{ }^{2}$ The appropriate diabatic state calculations for these configurations in $\mathrm{O}_{2}$ and $\mathrm{N}_{2}$ have, unfortunately, not been reported. Nevertheless, it can be expected that the $\left(1 \pi_{u}^{-1} \delta_{g} 3 d\right)^{3} \Pi_{u}$ diabatic state, which should correlate with the dipole allowed $\left(2 p^{-1} 3 d\right)^{3} D^{0}$ atomic oxygen state, crosses the outer limbs of the $n d \delta_{\varepsilon}$ Rydberg series (Table IV) and becomes a photoionization resonance at the ground-state equilibrium internuclear separation. The $1 \pi_{u} \rightarrow k \sigma_{s}$ profile is evidently monotonic, and, of course, joins smoothly to the Stieltjes histogram in the discrete spectral interval, ${ }^{35}$ which is dominanted by the strong $1 \pi_{u} \rightarrow 3 s \sigma_{s}$ Rydberg transition (Table IV). Again, a similar behavior is seen in the corresponding profile in $\mathrm{N}_{2}{ }^{2}$ By contrast, the $1 \pi_{u} \rightarrow k \pi_{\varepsilon}$ profile in $\mathrm{N}_{2}$ shows a strong peak near threshold, whereas the present $1 \pi_{u} \rightarrow k \pi_{s}$ cross section in $\mathrm{O}_{2}$ [Fig. 2(a)] is flat and broad, with no evidence of a large contribution near threshold. As indicated above, re-examination of the $\mathrm{N}_{2}$ calculations suggests that the large threshold value is a consequence of a $\pi \rightarrow \pi^{*}$ contribution. When appropriate projection procedures are employed in $\mathrm{N}_{2}$ to localize the $\pi \rightarrow \pi^{*}$ contribution, a $1 \pi_{u} \rightarrow k \pi_{g}$ photoionization profile similar to that of Fig. 2(a) is obtained. ${ }^{57}$ Recall that the strong $1 \pi_{u}-1 \pi_{f}$ intravalence transition is not included in the present calculations, thereby eliminating its possible contamination of the continuum. Because four parent $\mathrm{O}_{2}^{+}$ionic states $\left[a^{4} \Pi_{u}, A^{2} \Pi_{u}, 2^{2} \Pi_{u}, 3^{2} \Pi_{u}\right]$ are formed upon removal of a $1 \pi_{u}$ electron, comparison with experiment must be made with care. It is found that when the measured $a^{4} \Pi_{u}$ and $A^{2} \Pi_{u}$ contributions, which are not separately resolved, are combined with the measured $2^{2} \Pi_{u}$ and $3^{2} \Pi_{u}$ contributions, the latter being shifted to the $a^{4} \Pi_{u}$ threshold, the theoretical and experimental results are in good agreement. Moreover, the three separately measured cross sections $\left[a^{4} \Pi_{u}+A^{2} \Pi_{u}, 2^{2} \Pi_{u}, 3{ }^{2} \Pi_{u}\right]$ exhibit peaks $\sim 5 \mathrm{eV}$ above their respective thresholds, providing substantial support for the presence of the predicted $1 \pi_{u}-k \delta_{\varepsilon} 3 d$ resonance feature.

The appearance of a prominent resonancelike $\sigma_{u} 2 p$ 
feature in the $3 \sigma_{z}-k \sigma_{u}$ cross section of Fig. 3(a) is in complete accord with the results of the previous investigations in $\mathrm{N}_{2}$ and $\mathrm{CO},{ }^{1-3}$ with the foregoing remarks made in connection with the $1 \pi_{g} \rightarrow k \sigma_{u}$ channel, and with the observation that the discrete $3 \sigma_{g} \rightarrow n \sigma_{u}$ series (Table $V)$ does not include an intravalence contribution. Moreover, the valence-basis CI calculations (Table VI) predict the presence of two diabatic states, corresponding to $b^{4} \Sigma_{p}^{-}$and $B^{2} \Sigma_{\boldsymbol{g}}^{-}$parentages, at the appropriate resonance position. Finally, the presence of a prominent feature in the $\mathrm{O}_{2} K$-edge cross section just above threshold, which can be given a $1 \sigma_{g}-k \sigma_{u}$ assignment, provides additional support for the appearance of a $\sigma_{u} 2 p$ contribution in the photoionization continuum. ${ }^{24}$

Although the experimentally determined partial-channel cross sections for $1 \pi_{s}, 1 \pi_{u}$, and $3 \sigma_{g}$ photoionization in $\mathrm{O}_{2}$ do not provide unambiguous verification of the calculated resonance features, the measured values are certainly compatible with the theoretical results and with the corresponding interpretations. Moreover, the independent measurements of the total photoabsorption and ionization cross sections shown in Fig. 4 are also compatible with the present calculations, and perhaps also provide some support for the $3 \sigma_{z} \rightarrow k \sigma_{u} 2 p$ resonancelike feature slightly above the corresponding threshold. ${ }^{60}$

\section{CONCLUDING REMARKS}

The qualitative discussions of electronic excitation and ionization processes presented here, and the quantitative separated-channel, static-exchange calculations upon which they are based, attempt to provide an overall elementary account of both the discrete and continuous portions of the valence spectrum in molecular oxygen. Use of the Stieltjes-Tchebycheff technique and $L^{2}$ calculations allows rather straightforward application of familiar molecular-orbital concepts to the photoionization continua corresponding to various occupied orbitals, and thereby provides a basis for discussing the discrete and continuous excitations from a common perspective. Most important, the concepts of intense int ravalencelike transitions on the one hand, and generally weaker Rydberg-like transitions on the other, are seen to carry over in a natural way into the photoionization continua. In particular, the $1 \pi_{\text {g }}$ photoionization continuum in oxygen is seen to be dominated by features that are conveniently regarded as arising from the presence of $\pi_{u} 3 d, \delta_{u} 3 d$, and $\sigma_{u} 2 p$ final-state resonancelike orbitals in the $k \pi_{u}$, $k \delta_{u}$, and $k \sigma_{u}$ channels, respectively. Similarly, the $1 \pi_{u}$ and $3 \sigma_{q}$ cross sections contain $\delta_{q} 3 d$ and $\sigma_{u} 2 p$ resonancelike features, respectively. These final-state valence orbitals do not contribute to the corresponding discrete spectral regions, but rather are associated with diabatic states that cross the outer limbs of the various Rydberg series as functions of internuclear distance, becoming photoionization resonance above the appropriate ioniza tion potentials at the ground-state equilibrium separation.

In addition to providing an overall descriptive account of the electronic dipole excitation spectrum in molecular oxygen, the calculations reported here are seen to be in generally good quantitative accord with very recent corresponding experimental studies. Some care is required in making comparisons between theory and experiment in molecular oxygen, particularly since there are four final ionic states and corresponding cross sections associated with the removal of a $1 \pi_{u}$ electron. Although the measured photoelectron spectra and partial-channel cross sections are relatively complex, good agreement between theory and experiment obtains when proper cognizance is taken of the ionic parentages of the various multiplet states. Although further computational refinements, including coupled-channel, static-exchange calculations, allowance for core polarization and relaxation, explicit treatments of vibrational degrees of freedom, and of aspects of autoionizing states, will improve agreement with experiment, it is clear that the general approach employed here should continue to provide useful first approximations to discrete and continuous dipole excitation spectra in small molecules. Further computational applications are consequently in progress and will be reported subsequently.

\section{ACKNOWLEDGMENTS}

The authors thank C. E. Brion and M. J. Van der Wiel for kindly making the results of their experimental studies available prior to publication, and for helpful correspondence. We also thank R. L. Blake for permission to cite his experimental results prior to publication, and A. U. Hazi for his help in clarifying the origins of the four $1 \pi_{u}$ cross sections. Acknowledgment is made to the National Science Foundation for support provided to B.V.M., and to the Donors of the Petroleum Research Fund, administered by the American Chemical Society, and to the National Research Council, for providing support to P.W.L. The kind hospitality of J. O. Arnold and S. R. Langhoff of the NASA-Ames Research Center Computational Chemistry Group, and of D. Bershader of the Department of Aeronautics and Astronautics, Stanford University, to P. W. L. is also gratefully acknowledged.

${ }^{1}$ T. N. Rescigno and P. W. Langhoff, Chem. Phys. Lett. 51, 65 (1977).

${ }^{2}$ T. N. Rescigno, C. F. Bender, B. V. McKoy, and P. W. Langhoff, J. Chem. Phys. 68, 970 (1978).

${ }^{3}$ N. Padial, G. Csanak, B. V. McKoy, and P. W. Langhoff, J. Chem. Phys. 69, 2992 (1978).

${ }^{4}$ P. W. Langhoff, S. R. Langhoff, and C. T. Corcoran, J. Chem. Phys. 67, 1722 (1977).

${ }^{5}$ P. W. Langhoff, A. E. Orel, T. N. Rescigno, and B. V. McKoy, J. Chem. Phys. 69, 4689 (1978).

${ }^{6}$ Geoffry R. J. Williams and P. W. Langhoff, Chem. Phys. Lett. 60, 201 (1978).

${ }^{7} \mathrm{~J}$. A. R. Samson and J. L. Gardner, J. Electron Spectrosc. Relat. Phenom. 8, 35 (1976).

${ }^{8}$ A. Hamnett, W. Stoll, and C. E. Brion, J. Electron Spectrosc. Relat. Phenom. 8, 367 (1976).

${ }^{9}$ G. R. Wight, M. J. Van der Wiel, and C. E. Brion, J. Phys. B 9, 675 (1976).

${ }^{10} \mathrm{E}$. W. Plummer, T. Gustafsson, W. Gudat, and D. E. Eastman, Phys. Rev. A 15, 2339 (1977).

${ }^{11} \mathrm{~J}$. A. R. Samson, G. N. Haddad, and J. L. Gardner, J. Phys. B 10, 1749 (1977).

${ }^{12}$ R. B. Kay, Ph. E. Van der Leeuw, and M. J. Van der Wiel, J. Phys. B 10, 2513, 2521 (1977). 
${ }^{13}$ P. R. Woodruff and G. V. Marr, J. Phys. B 9, L377 (1976); Proc. R. Soc. London Ser. A 358, 87 (1977).

${ }^{14}$ G. V. Marr, J. M. Morton, R. M. Holmes, and D. G. McCoy, J. Phys. B (to be published).

${ }^{15}$ J. L. Dehmer and D. Dill, Phys. Rev. Lett. 35, 213 (1975);

J. Chem. Phys, 65, 5327 (1976).

${ }^{16}$ J. W. Davenport, Phys. Rev. Lett. 36, 945 (1976); Int. J. Quantum Chem. Symp. 11, 89 (1977).

${ }^{17}$ J. -T. J. Huang, F. O. Ellison, and J. W. Rabalais, J. Electron Spectrosc. Relat. Phenom. 3, 339 (1974).

${ }^{18}$ H. C. Tuckwell, J. Phys. B 3, 293 (1970).

${ }^{19} \mathrm{~F}$. Hirota, J. Electron Spectrosc. Relat. Phenom. 9, 149 (1976).

${ }^{20}$ R. D. Hudson (Ed.) "Critical Review of Ultraviolet Photoabsorption Cross Sections for Molecules of Astrophysical and Aeronomic Interest," Natl. Stand. Ref. Data Ser. Natl. Bur. Stand. 38 (1971).

${ }^{21}$ P. H. Krupenie, J. Phys. Chem. Ref. Data 1, 423 (1972).

${ }^{22}$ G. R. Wight and C. E. Brion, J. Electron Spectrose. Relat. Phenom. 4, 313 (1974); A. P. Hitchcock and C. E. Brion, J. Phys. B (to be published).

${ }^{23}$ R. E. LaVilla, J. Chem. Phys. 63, 2733 (1975).

${ }^{24}$ D. M. Barrus, R. L. Blake, A. J. Burek, K. C. Chambers, and A. L. Pregenzer, Phys. Rev. (to be published).

${ }^{25}$ J. A. R. Samson, J. L. Gardner, and G. N. Haddad, J. Electron Spectrosc. Relat. Phenom. 12, 281 (1977).

${ }^{26}$ G. Mehlman, D. L. Ederer, and E. B. Saloman, J. Chem. Phys. 88, 1862 (1978). See also L. C. Lee, R. W. Carlson, D. L. Judge, and M. Ogawa, J. Quant. Spectrosc. Radiat. Transfer 13, 1023 (1973).

${ }^{27}$ D. G. McCoy, J. M. Morton, and G. V. Marr, J. Phys. B (to be published).

${ }^{28}$ C. E. Brion, K. H. Tan, M. J. Van der Wiel, and Ph. E. Van der Leeuw, J. Electron Spectrosc. Relat. Phenom. 17, 101 (1979).

${ }^{29}$ P. W. Langhoff, C. T. Corcoran, J. S. Sims, F. Weinhold, and R. M. Glover, Phys. Rev. A 14, 1042 (1976).

${ }^{30} \mathrm{C}$. T. Corcoran and P. W. Langhoff, J. Math. Phys. 18, 651 (1977).

${ }^{31}$ P. W. Langhoff, Int. J. Quantum Chem. Symp. 8, 347 (1974); 11,301 (1977).

${ }^{32}$ P. W. Langhoff, "The Stieltjes-Tchebycheff Approach to Molecular Photoionization Studies," in Electron - and PhotonMolecule Collisions, edited by T.N. Rescigno, B. V. McKoy, and B. Schneider (Plenum, New York, 1979), pp. 183-224.

${ }^{33} \mathrm{H}$. F. Schaefer III, The Electronic Structure of Atoms and Molecules (Addison-Wesley, Reading, MA, 1972); Ann. Rev. Phys. Chem. 27, 261 (1976).

${ }^{34} \mathrm{~T}$. H. Dunning and P. J. Hay, in Modem Theoretical Chemistry, edited by H. F. Schaefer, III (Plenum, New York, 1976), Vol. 3, Chap. 1 .

${ }^{35} \mathrm{P}$. W. Langhoff and C. T. Corcoran, J. Chem. Phys. 61, 146 (1974).

${ }^{36}$ D. W. Turner, C. Baker, A. D. Baker, and C. R. Brundle, Molecular Photoelectron Spcctroscopy (Wiley, New York,
1970).

${ }^{37} \mathrm{~K}$. Siegbahn, C. Nordling, G. Johansson, J. Hedman, P. F. Hedén, K. Hamrin, U. Gelius, T. Bergmark, L. O. Werme, R. Manne, and Y. Bauer, ESCA Applied to Free Molecules (North-Holland, Amsterdam, 1969).

${ }^{38} \mathrm{~J}$. Raffery and W. G. Richards, Int. J. Mass Spectrom. Ion Phys. 6, 269 (1971); O. Edqvist, E. Lindholm, L. E. Selin, and L. Åsbrink, Phys. Scr. 1, 25 (1970).

${ }^{39}$ P. E. Cade and A. C. Wahl, At. Data Nucl. Data Tables 13, 339 (1974).

${ }^{40}$ F. R. Gilmore, J. Quant. Spectrosc. Radiat. Transfer. 5, 369 (1965).

${ }^{41} \mathrm{~J}$. B. Rose and V. McKoy, J. Chem. Phys. 55, 5435 (1971).

${ }^{42} \mathrm{H}$. Lefebvre-Brion, C. Moser, and R. K. Nesbet, J. Mol. Spectrosc. 13, 418 (1964).

${ }^{43} \mathrm{E}$. U. Condon and G. H. Shortley, The Theory of Atomic Spectra (Cambridge U. P., London, 1963), p. 98.

${ }^{44} \mathrm{G}$. Herzberg, Spectra of Diatomic Molecules (Van Nostrand, New York, 1950), 2nd ed.

${ }^{45} \mathrm{~K}$. Tanaka and M. Yoshimine, J. Chem. Phys. 70, 1626 (1979).

${ }^{46}$ R. S. Mulliken and C. A. Rieke, Rep. Prog. Phys, 8, 321 (1941).

${ }^{47}$ R. S. Mulliken and W. C. Ermler, Diatomic Molecules (Academic, New York, 1977).

${ }^{48} \mathrm{O}$. Sinanoğlu, "Theory of Intravalency and Rydberg Transitions in Molecules," in Chemical Spectroscopy and Photochemistry in the Vacuum Ultravialet, edited by C. Sandorfy, P. J. Ausloss, and M. B. Robin (Reidel, Boston, MA, 1974), pp. 337-384.

${ }^{49} \mathrm{Y}$. Tanaka and T. Takamine, Tokyo Inst. Phys. Chem. Res. 39, 437 (1942).

${ }^{50}$ D. C. Cartwright, W. J. Hunt, W. Williams, S. Trajmar, and W. A. Goddard, J. Chem. Phys. 8, 2436 (1973).

${ }^{51}$ E. Lindholm, A rk. Fys. 40, 117 (1969).

${ }^{52} \mathrm{~K}$. Morokuma and H. Konishi, J. Chem. Phys. 55, 402 (1971).

${ }^{53}$ H. F. Schaefer, III and W. H. Miller, J. Chem. Phys. 55, 4107 (1971).

${ }^{54}$ R. J. Buenker and S. D. Peyerimhoff, Chem. Phys. 8, 324 (1975); Chem. Phys. Lett. 34, 225 (1975).

${ }^{55}$ R. P. Saxon and B. Liu, J. Chem. Phys. 67, 5432 (1977).

${ }^{56}$ P. W. Langhoff, A. Gerwer, C. Asaro, and B. V. McKoy, Int. J. Quantum Chem. Symp. (to be published).

${ }^{57}$ T. N. Rescigno, A. Gerwer, B. V. McKoy, and P. W. Langhoff, Chem. Phys. Lett. 66, 116 (1979).

${ }^{58} \mathrm{~J}$. Leclercq, Ann. Astrophys. 30, 93 (1967).

${ }^{59}$ D. N. Dixon and S. E. Hull, Chem. Phys. Lett. 3, 367 (1969).

${ }^{60} \mathrm{~T}$. Gustafsson (private communication) has recently completed high-resolution PES and partial-channel photoionization cross section studies in $\mathrm{O}_{2}$ the results of which are in excellent accord with the present calculations, and which, in particular, verify the existence of the $3 \sigma_{g}-k \sigma_{u} \Sigma p$ resonance just above threshold. We thank Dr. Gustafsson for making his results available prior to publication. 